\title{
Muscle group specific transcriptomic and DNA methylation differences related to developmental patterning in FSHD
}

Katherine Williams ${ }^{1,2}$, Xiangduo Kong ${ }^{3}$, Nam Viet Nguyen ${ }^{3}$, Cassandra McGill ${ }^{1}$, Rabi Tawil $^{4}$, Kyoko Yokomori ${ }^{3}$, Ali Mortazavi ${ }^{1,2}$

1) Department of Developmental and Cell Biology, University of California Irvine, Irvine, California, United States of America, 2) Center for Complex Biological Systems, University of California Irvine, Irvine, California, United States of America, 3) Department of Biological Chemistry, School of Medicine, University of California Irvine, Irvine, California, United States of America, 4) Neuromuscular Disease Unit, Department of Neurology, University of Rochester Medical Center, Rochester, New York, United States of America

*kyokomor@uci.edu (KY); ali.mortazavi@uci.edu (AM) 


\section{Abstract}

Muscle groups throughout the body are specialized in function and are specified during

3 development by position specific gene regulatory networks. In developed tissue, myopathies

4 affect muscle groups differently. Facioscapulohumeral muscular dystrophy, FSHD, affects upper

5 body and tibialis anterior (TA) muscles earlier and more severely than others such as quadriceps.

6 To investigate an epigenetic basis for susceptibility of certain muscle groups to disease, we

7 perform DNA methylation and RNA sequencing on primary patient derived myoblasts from TA

8 and quadricep for both control and FSHD2 as well as RNA-seq for myoblasts from FSHD1

9 deltoid, bicep and TA over a time course of differentiation. We find that TA and quadricep retain

10 methylation and expression differences in transcription factors that are key to muscle group

11 specification during embryogenesis. FSHD2 patients have differences in DNA methylation and

12 expression related to SMCHD1 mutations and FGF signaling. Genes induced specifically in

13 FSHD are more highly expressed in commonly affected muscle groups. We find a set of genes

14 that distinguish more susceptible muscle groups including development-associated TFs and

15 genes involved in WNT signaling. Adult muscle groups therefore retain transcriptional and DNA

16 methylation differences associated with development, which may contribute to susceptibility in

17 FSHD.

\section{Background}

The human body has over 650 named skeletal muscle groups [1,2]. These groups are

21 heterogeneous in terms of compositions of fast and slow twitch fibers and regenerative

22 capabilities [3]. Initial muscle cell specification through the activation of the key myogenic

23 factors MRF4/MYF5, MYOD, and MYOG is regulated by upstream transcription factors that 
24 vary depending on the location of the muscle cells [4,5]. PITX2 plays a central role in regulating

25 MYF5/MRF4 in extraocular muscles and can regulate MYOD in limb muscles [4-7]. SIX and

26 EYA TFs activate PAX3 and MYF5/MRF4 in limb muscles [7].

The limbs are initially specified by HOX genes which activate TBX5 in the forelimb and

29 PITX1 in the hindlimb [8]. PITX1 then activates TBX4 in the hindlimb [8]. TBX5 and TBX4

30 activate FGF10 which forms a gradient with FGF8 that is expressed at the apical ectodermal

31 ridge to control limb outgrowth [8]. Limb outgrowth specifies the proximal/distal axis resulting

32 in three major regions; the stylopod, zeugopod and autopod [8]. MEIS factors are expressed

33 proximally in the stylopod but are repressed by SHOX2 distally into the zeugopod where

34 HOXA11 is expressed [9,10]. Dorsal/ventral patterning is controlled by WNT and LMX1B

35 expression on the dorsal side and EN1 on the ventral side which represses WNT [11].

Gene expression in embryonic development of different muscle groups is well studied,

38 but few studies have surveyed gene expression in adult tissue [12,13]. An estimated 50\% of

39 transcripts are differentially expressed in adult muscle with some heterogeneity coming from

40 fiber-type composition and expression of developmental related genes [14]. In addition to

41 transcription, adult muscle groups retain DNA methylation differences [15]. The molecular

42 differences between muscle groups may contribute to severity of affectedness in myopathies.

Many myopathies affect muscle groups of the body exclusively or more severely, such as

45 facioscapulohumeral muscular dystrophy (FSHD), which is characterized by noticeable

46 weakness in the most commonly affected muscle groups in the upper body including facial and 
47 humeral $[16,17]$. FSHD progression into muscle groups is sporadic, but some groups such as the

48 tibialis anterior are more commonly affected or affected earlier [18]. Certain muscle groups are

49 less frequently affected or affected later including the quadricep and the deltoid [19,20].

FSHD is caused by the misexpression of the embryonic transcription factor $D U X 4$ in

52 skeletal muscle [17,19]. DUX4 activates expression of its target genes including a number of

53 embryonic related transcription factors and chromatin remodelers as well as repeat elements

54 including endogenous retroviruses such as ERVLs [21-24]. DUX4 target gene expression

55 correlates with signs of active disease $[25,26]$. Comparison of the commonly affected bicep to

56 the less affected deltoid found greater expression differences between FSHD and control in bicep

57 than deltoid but did not assess differences in DUX4 target gene expression between the two

58 muscle groups [27].

We survey transcription and DNA methylation to establish differences between muscle

61 groups that may contribute to susceptibility in FSHD. We perform RNA-seq and probe enriched

62 bisulfite sequencing to survey transcriptional and DNA methylation differences between tibialis

63 anterior (TA) and quadricep in myoblasts from healthy patients and those with FSHD2 with

64 SMCHD1 mutations. We examine differences in DNA methylation and expression between TA

65 and quadricep, and find retained differences in TFs important for development. Next, we find a

66 set of genes specifically upregulated in FSHD2 over time including DUX4 target genes. Notably,

67 the promoters of these genes are highly methylated in both FSHD2 and control cells. We look at

68 genome-wide DNA methylation and gene expression differences in FSHD2 and find

69 hypomethylation and increased expression for genes in regions regulated by SMCHD1. We 
70 survey differences for TE loci and find repeat elements upregulated in response to DUX4 are

71 especially upregulated in FSHD2 cells from TA but not quadricep. To determine possible

72 differences in gene expression correlating with susceptibility, we performed RNA-seq for

73 myoblasts from the TA, bicep and deltoid of FSHD1 patients. We identify muscle group specific

74 gene expression for TA, quadricep, bicep and deltoid, and genes differentially expressed between

75 muscle groups with different susceptibilities to FSHD.

$77 \quad$ Results

Muscle group specific DNA methylation and gene expression in developmental TFs

To identify epigenetic differences between muscle groups, we performed capture

81 enrichment bisulfite sequencing (Methods) on two primary myoblast cell lines from quadricep

82 and two from tibialis anterior (TA) for days 0, 3 and 12 of differentiation (Figure S1). We

83 merged CpG sites within 200 bp into regions which were then filtered for coverage (Methods).

84 We found very few differentially methylated regions (DMRs) (percent diff 25, q-value $<0.01$ )

85 between the differentiation days and decided to treat the different days as replicates for further

86 comparisons (Figure S2A, S2B).

We compared TA and quadricep and found 1081 regions more highly methylated in

89 quadricep as well as 686 regions with higher methylation in TA (Figure 1A, S2B). The regions

90 with the highest percent methylation differences are associated with transcription factors that

91 play a role in limb specific muscle specification. These include PITX2, which controls location

92 specific gene networks to induce MRFs [4]. Also included is MEIS1, which is expressed in the 
93 stylopod and is more highly methylated in TA than quad (Figure 1A) [9]. Of the 1767 DMRs,

94357 are associated with 265 TFs, and 83 of those are involved in pattern specification while 39

95 are involved in muscle structure development (Figure S2C, Table S1). In support of this, gene

96 ontology analysis for these DMRs revealed significant association with development and

97 structure morphogenesis (Figure 1B). Quadricep and TA therefore retain DNA methylation

98 patterns from their specification.

99

A

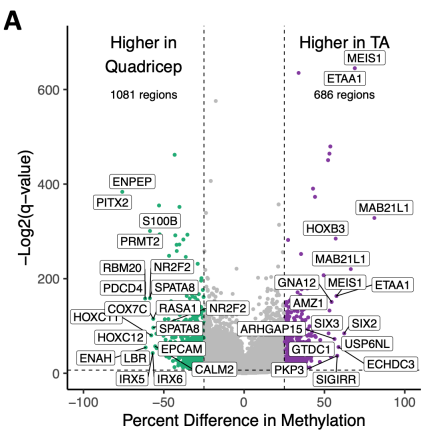

D

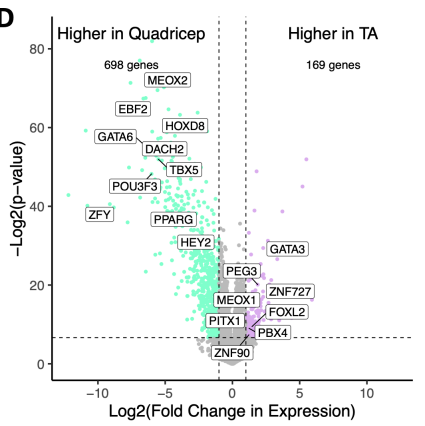

B
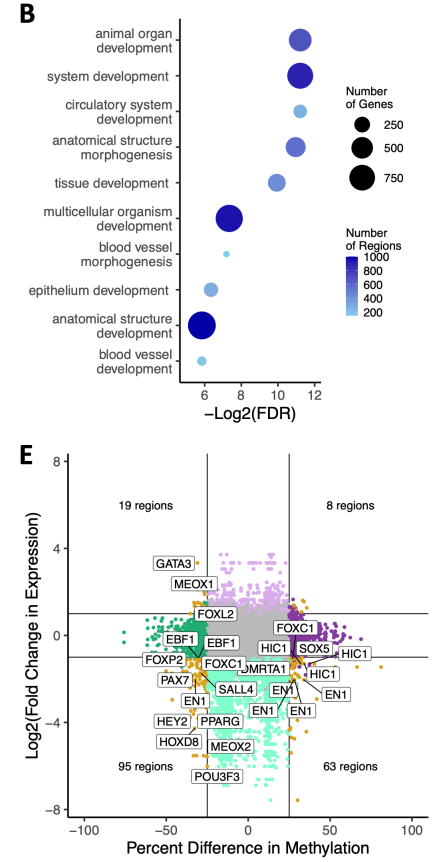

C

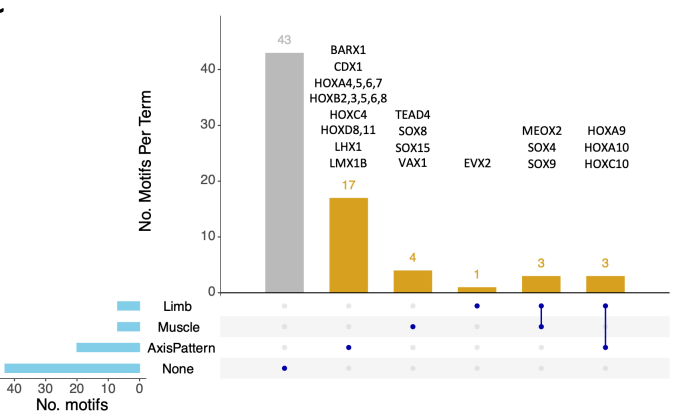

$\mathbf{F}$

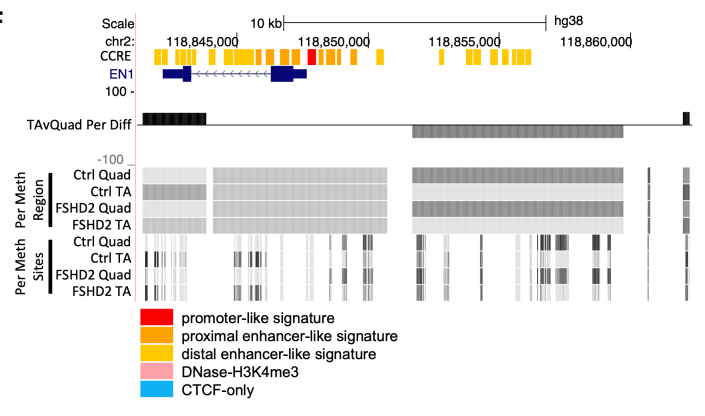

101 Figure 1: Adult muscle cells retain differences in DNA methylation and gene expression for

102 transcription factors involved in developmental patterning (A) Volcano plot of DNA

103 methylation differences for control cells from TA versus quadricep. Regions with a p-value of

$104>0.01$ or a percent methylation difference of $<25$ are taken as not significant and colored in grey.

105 Regions with more methylation in TA are in purple. Regions with more methylation in quadricep

106 are in green. The top 10 regions with the highest percent difference in the positive or negative

107 directions are labelled. The numbers of regions higher in TA and quadricep are labelled at the 
108 top. Vertical lines intersect y-axis at -25 and 25 percent. Horizontal line intersects y-axis at -

$109 \log 2(0.01)$. (B) Gene ontology terms enriched in differentially methylated regions between TA

110 and quadricep. The top 10 significant terms ( $p$-value $<0.05)$ with at least 10 genes associated to

111 the term from the background set of regions detected are shown. Color indicates number of

112 differentially methylated regions in the term. Size indicates the number of genes associated with

113 the differentially methylated regions in the term. (C) Upset plot of gene ontology terms of

114 transcription factors whose motifs are enriched in differentially methylated regions between TA

115 and quadricep. Each transcription factor was annotated with GO terms related to limb, muscle or

116 axis patterning (see Methods for details). The number of transcription factors found enriched in

117 the DMRs that falls into the individual categories are shown in blue on the left. The number of

118 transcription factors belonging to the interest of the categories indicated in dark blue are in gold

119 and grey on the top. (D) Volcano plot of gene expression differences for control cells from TA

120 versus quadricep. Genes with a $p$-value of $>0.01$ or an absolute $\log 2$ (fold change in expression)

$121<1$ are in grey. Genes with higher expression in the TA are in light purple. Genes with higher

122 expression in the quadricep are in light green. The numbers of genes with higher expression in

123 TA or quadricep are labelled at the top. The 10 transcription factors with the lowest p-values for

124 positive or negative fold change are labelled. Vertical lines intersect $y$-axis at -1 and $1 \log 2$ (fold

125 change). Horizontal line intersects y-axis at - $\log 2(0.01)$. (E) Scatterplot of differences in DNA

126 methylation and expression of nearby gene for TA versus quadricep. Regions are associated with

127 genes through GREAT. Regions with significant differences in both DNA methylation and gene

128 expression are in gold, and the numbers of these regions are labelled in the corresponding

129 quadrant. All regions associated with transcription factors in the given quadrants are labelled.

130 Regions associated with genes with significant differences in gene expression but not DNA 
131 methylation are colored in light purple and light green. Regions with significant differences in

132 DNA methylation but not in expression of the associated gene are colored in green and purple.

133 Vertical lines intersect $\mathrm{x}$-axis at -25 and 25 percent. Horizontal lines intersect $\mathrm{y}$-axis at -1 and 1

$134 \log 2$ (fold change). (F) UCSC genome browser shot of DNA methylation near EN1. EN1 gene

135 model is from gencode v28. Percent methylation for each group of samples in the merged region

136 are labelled with increase in methylation indicated with darker grey. The percent difference in

137 methylation is indicated as a barplot with higher methylation in TA as positive and higher

138 methylation in quadricep as negative. Percent methylation at individual sites for the given groups

139 are at the bottom with darker color indicating higher methylation.

We looked for TF motifs enriched in the DMRs between TA and quadricep to see what

143 could be regulating these regions. The DMRs are enriched for motifs of developmental

144 transcription factors (Figure S2D). Importantly, we found motifs for key transcription factors

145 involved in axis specification, skeletal muscle development and differentiation, and limb

146 specification and morphogenesis, including motifs for 19 TFs controlling anterior/posterior,

$147 \mathrm{dorsal} /$ ventral and/or proximal/distal pattern specification; six HOXA (HOXA4, 5, 6, 7, 9, 10),

148 five $\operatorname{HOXB}(\mathrm{HOXB} 2,3,5,6,8)$, two $\mathrm{HOXC}(\mathrm{HOXC4}, 10)$, two $\mathrm{HOXD}(\mathrm{HOXD} 8,11)$, as well

149 as CDX1, LMX1B, LHX1, and BARX1 (Figure 1C, S2D). Seven motifs are from TFs important

150 for limb specification, patterning or development, and seven motifs are from TFs involved in

151 muscle development or differentiation (Figure 1C, S2D). MEOX2 is involved in both muscle and

152 limb development when it acts in concert with PAX3 and SIX1/4 to activate MYF5 in myoblasts

153 migrating into the limb [28]. 
TFs are differentially expressed between the tissues. We performed RNA-seq on days 0 to 5 and

157 day 12 of differentiation for the two quadricep and two TA cell lines (Fig S1A). We found 867

158 genes differentially expressed $(|\log \mathrm{FC}|>1, \mathrm{FDR}<0.01)$ including 54 TFs (Figure 1D). Of these

159 TFs, 26 are related to axis patterning, 16 are involved in appendage development, and 12 are

160 involved in muscle organ development (Figure S2E). The hindlimb determining TF PITX1 is

161 more highly expressed in TA than quadricep (Figure 1D) [29]. PITX1 activates TBX4, which was

162 differentially methylated, in the developing hindlimb (Table S1) [8]. TBX5, which is responsible

163 for specifying the forelimb, is more highly expressed in the quadricep than the TA (Figure 1D)

164 [8]. Five of the differentially expressed TFs also had motifs which were significantly enriched in 165 the differentially methylated regions; CDX1, MEOX2, HOXD8, FOXC1 and FOXD2 (Table

$166 \mathrm{~S} 2)$. CDX1 is expressed in the limb bud and is responsive to retinoic acid (RA), WNT and FGF

167 signals [30,31]. HOXD8 is expressed during early patterning in the proximal limb [8].

We compared the differences in DNA methylation with changes in gene expression by

170 associating the differentially methylated regions with genes using GREAT (Methods) [32].

171 Seventeen of the TFs were both differentially expressed and differentially methylated (Figure

172 1E). This included EN1, which represses $W N T 7 A$ in dorsal/ventral patterning, and $S A L L 4$, which

173 regulates hindlimb outgrowth with TBX4 by regulating and FGF10 [11,33]. Differences in

174 methylation around EN1 included proximal regions and the gene body that overlap annotated

175 candidate cis-regulatory elements (Figure 1F). We compared the differentially expressed genes

176 in TA and quadricep from our primary myoblasts from control and FSHD patients to those from 
177 RNA-seq from TA and quadricep patient biopsy samples [25]. PITXI and IRX5 were upregulated

178 in TA in both cell lines and biopsy samples for FSHD and control (Figure S2F). IRX5 expression

179 in the hindlimb bud is important for specifying proximal and anterior regions of the limb [34].

180 Thus, muscle groups retain DNA methylation and expression differences in adult tissue based on

181 early patterning.

182

183 Genes induced upon myogenesis in FSHD are more highly expressed in more susceptible

184 muscle

185 We previously identified a set of genes induced in FSHD2 cells upon differentiation up to

186 day 5 (Williams \& Jiang 2020). To assess FSHD specific gene upregulation in different muscle

187 groups, we performed RNA-seq for both TA and quadricep from SMCHD1 mutated FSHD2. We

188 found a set of 74 genes that are upregulated in both TA and quadricep FSHD2 cell lines starting

189 around day 3 of differentiation (Figure 2A, 2B). This includes 47 of our original 54 FSHD-

190 induced genes and an additional 27 genes (Figure 2B, S3A). Some of the new genes include

191 previously identified DUX4 target genes such as PRAMEF10 (Figure S2A) [21]. These

192 additional genes appear to be more lowly expressed than the set we identified previously (Figure

193 S2B). Interestingly, the quadricep, which is less susceptible to FSHD, has lower expression of

194 these FSHD-induced genes (Figure 2A). However, when comparing FSHD biopsy samples from

195 TA and quadricep, these genes are not significantly higher in the TA (Figure S3C, S3D). 
A

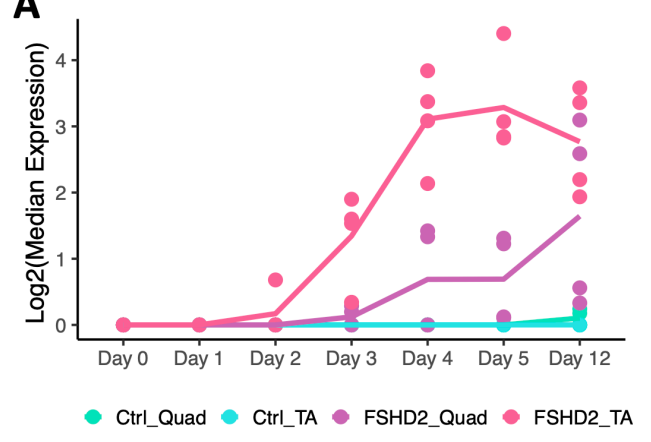

C

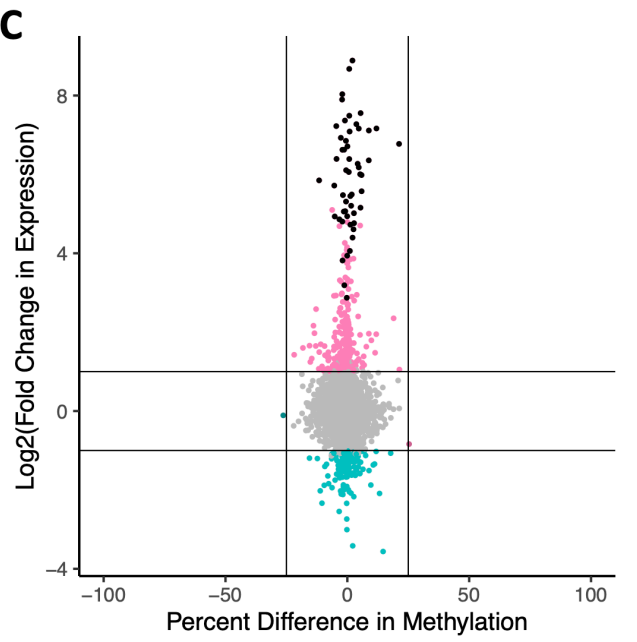

B

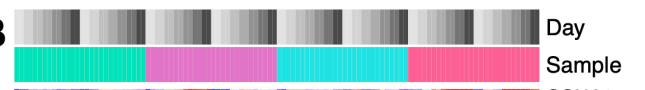

Day

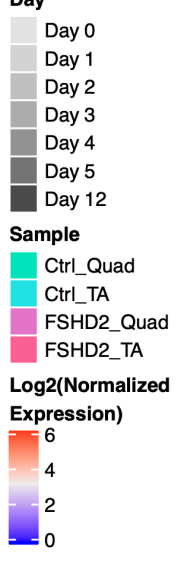

198 Figure 2: Promoters of FSHD-induced genes are not differentially methylated (A) Median

199 expression for given groups of 74 FSHD-induced genes with increased expression in FSHD2

200 during myogenesis. Dots represent median expression for individual samples. Line represents the

201 mean for the four samples in each group. (B) Heatmap of the 74 FSHD-induced genes ordered

202 by hierarchical clustering. (C) Scatterplot of differences in expression and promoter DNA

203 methylation for FSHD2 versus control. Promoters are defined as $-1.5 \mathrm{~kb}$ to $+0.5 \mathrm{~kb}$ around the

204 TSS. FSHD-induced genes are colored in black. Regions associated with genes with significant

205 differences in gene expression but not DNA methylation are colored in pink and teal. Regions

206 with significant differences in DNA methylation but not in expression of the associated gene are 
207 colored in dark teal and dark pink. Vertical lines intersect x-axis at -25 and 25 percent.

208 Horizontal lines intersect $y$-axis at -1 and $1 \log 2$ (fold change).

212 genes could partially explain their strong upregulation. We summarized the promoter CpG sites

213 for the 74 genes and recovered 53 gene promoters passing our coverage filters (Methods). The

214 promoters of these genes are not significantly differentially methylated despite substantial

215 increases in expression (Figure 2C). In fact, most of the promoters (33 out of 53) are highly

216 methylated with greater than 50\% methylation in both FSHD2 and control cell lines (Figure

217 S3E). Hypermethylation in the promoter is generally associated with repressing gene expression,

218 so we decided to determine whether DUX4 binds these promoter regions or could be regulating

219 expression through binding of other regulatory regions. Eleven of the highly methylated FSHD-

220 induced gene promoters overlap DUX4 binding sites determined by ChIP-seq (Figure S2D) [24].

To determine what TFs could be binding the methylated FSHD-induced gene promoters,

223 we looked for enrichment of unmethylated and methylated TF motifs determined by SELEX

224 [35]. While a methylated motif is not available for DUX4, the methylated motif for its paralog,

225 DUXA, is highly enriched in 41 of the 74 promoters (Figure S3F, Table S3). Additionally, the

226 methylated motif for OTX1, a PRD-like TF, is also enriched (Table S3). OTX1 has a similar

227 motif to the DUX4 target TF LEUTX [36]. Performing enrichment using canonical motifs only,

228 motifs for DUX4, DUXA, and OTX2, another PRD-like TF similar to LEUTX, are all enriched 
in the target promoters (Figure S3F, Table S3). The MEOX2 motif is also enriched, and MEOX2

\section{SMCHD1 mutation associated differences in DNA methylation and gene expression}

To assess global methylation differences between SMCHD1 mutated FSHD2 and control

234 cell lines, we compared all FSHD2 samples to all control samples and found 4527 regions more

235 highly methylated in control and 3542 in FSHD2 (percent diff $>10$, qvalue $<0.01$ ) (Figure $3 \mathrm{~A}$ ).

236 The top most differentially methylated region is $D B E T$, which is close to FRG2 and DUX4 in the

237 D4Z4 region on chromosome 4 that is hypomethylated in FSHD patients (Figure 3A) [37].

238 DBET is also less methylated in TA than quadricep in FSHD2 (Figure S4A). The D4Z4 region

239 on chromosome 10 near FRG2B and SYCE1 contains two hypomethylated regions in FSHD2,

240 and SYCE1 expression is upregulated in FSHD2 (Figure 3A, 3B) [38]. Hypomethylation in the

241 D4Z4 region on chromosome 10 has been shown to be FSHD2 specific, and the upregulation of

242 SYCE1 that we observe is not seen in FSHD1 (Figure S4B) [39,40]. SMCHD1 has been shown to

243 regulate clusters of genes such as the PCDH cluster on chromosome 5 (chr5:140,759,009-

244 141,523,383, hg38), the SNRPN cluster (chr15:23,548,232-23,697,319, hg38), the rRNA cluster

245 (chr1:228,552,374-228,653,525, hg38), and the tRNA cluster (chr1:161,395,860-161,624,746,

246 hg38) [41,42]. Out of 142 regions in the PCDH cluster and 6 in the rRNA cluster, we find 27

247 regions and 3 regions, respectively, with hypomethylation in FSHD2 compared to control

248 (Figure 3A). The SNRPN and tRNA clusters have negligible methylation differences (Figure 3A,

249 Table S4). The hypomethylation observed in the PCDH and rRNA clusters is low (less than

$25025 \%$ ) suggesting differences in methylation due to SMCHD1 heterozygosity are mild, especially

251 when compared with methylation differences near D4Z4 on chromosomes 4 and 10. 
A
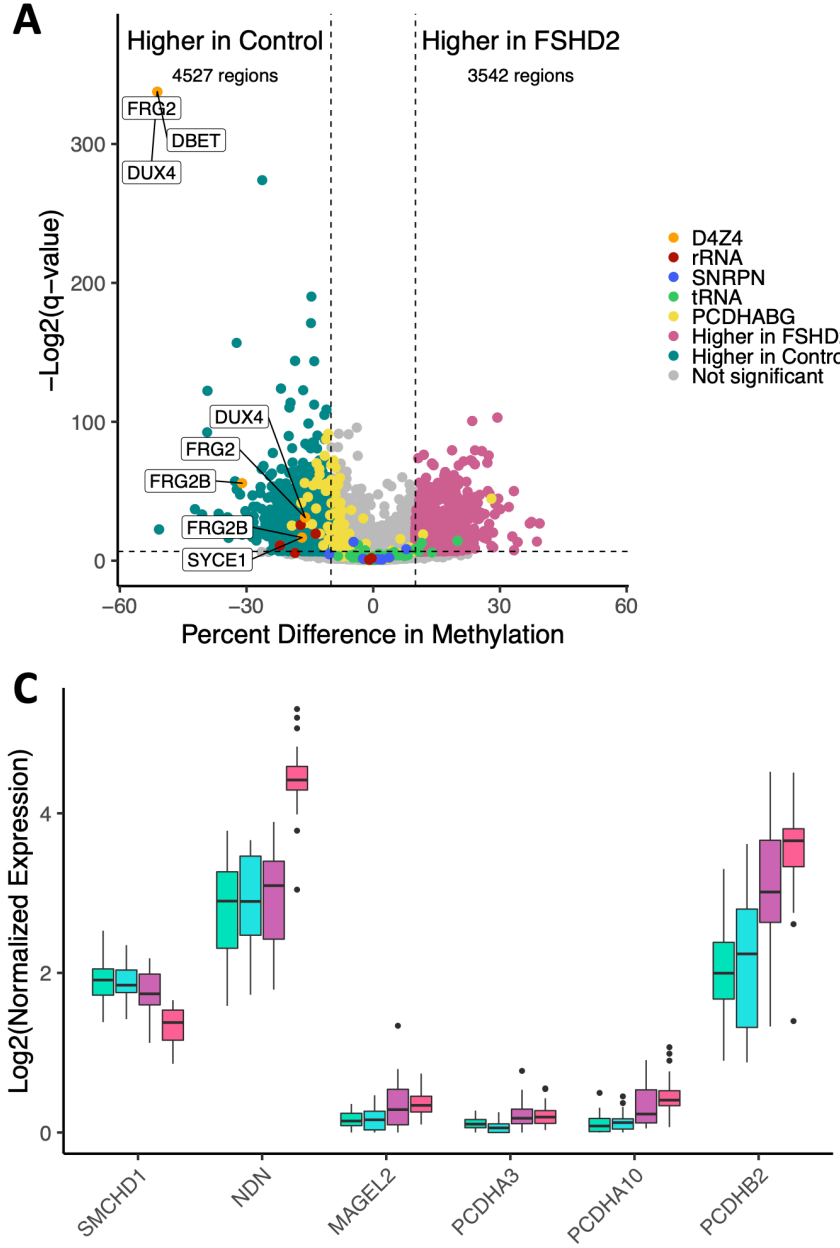

追 Ctrl_Quad 白 Ctrl_TA 追 FSHD2_Quad 追 FSHD2_TA

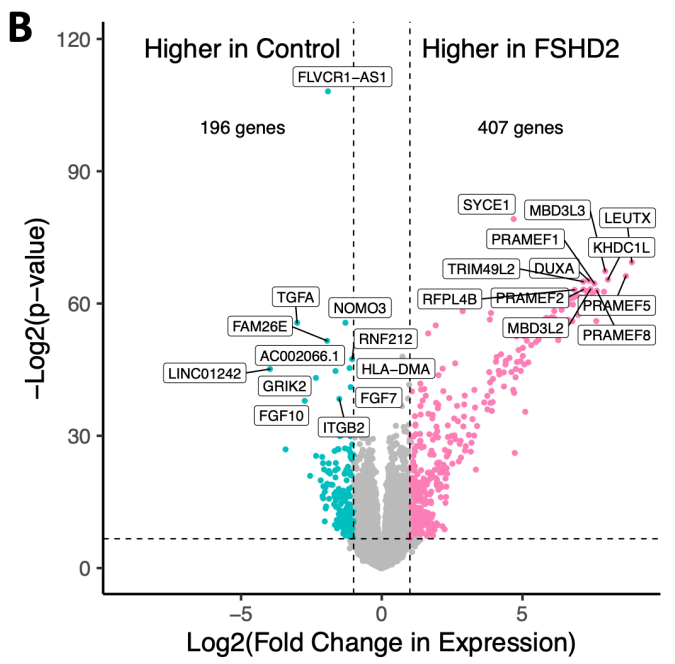

D

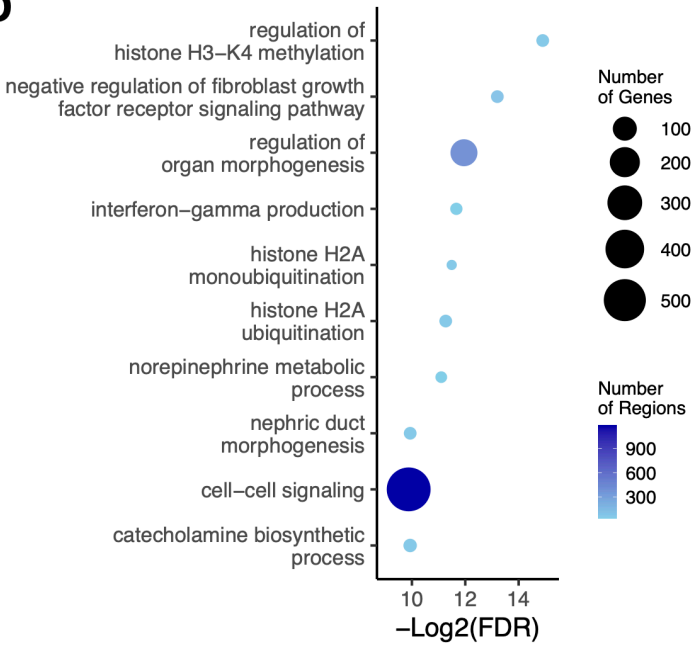

254 Figure 3: Global DNA methylation and gene expression differences in FSHD2 are

methylation differences for FSHD2 versus control. Regions with a $p$-value of $>0.01$ or a percent

methylation difference of $<10$ are taken as not significant and colored in grey. Regions with

more methylation in FSHD2 are in dark pink. Regions with more methylation in quadricep are in

259 dark teal. Regions within SMCHD1 regulated regions are colored according to the legend.

260 Regions in the D4Z4 region on chromosome 4 or 12 have associated genes labelled. The

261 numbers of regions higher in FSHD2 and control are labelled at the top. Vertical lines intersect 
$\mathrm{y}$-axis at -10 and 10 percent. Horizontal line intersects y-axis at $-\log 2(0.01)$. (B) Volcano plot of gene expression differences for FSHD2 versus control. Genes with a p-value of $>0.01$ or an

264 absolute $\log 2$ (fold change in expression) $<1$ are in grey. Genes with higher expression in the TA

265 are in pink. Genes with higher expression in the quadricep are in teal. The numbers of genes with

266 higher expression in FSHD2 or control are labelled at the top. The 12 genes with the lowest p-

267 values for positive or negative fold change are labelled. Vertical lines intersect y-axis at -1 and 1

$268 \log 2$ (fold change). Horizontal line intersects y-axis at $-\log 2(0.01)$. (C) Boxplot of expression for

269 genes in SMCHD1 regulated clusters and SMCHD1. (D) Gene ontology terms enriched in

270 differentially methylated regions between FSHD2 and control. The top 10 significant terms (p-

271 value $<0.05$ ) with at least 10 genes associated to the term from the background set of regions

272 detected are shown. Color indicates number of differentially methylated regions in the term. Size

273 indicates the number of genes associated with the differentially methylated regions in the term.

We looked at expression of genes in the PCDH and SNRPN clusters and found slight

277 upregulation in FSHD2 samples (Figure 3C). Despite not having significant methylation

278 differences, $N D N$ and MAGEL2 from the SNRPN cluster both have slightly elevated expression

279 in FSHD2 (Figure 3C). Interestingly, $N D N$ expression is much higher in FSHD2 cells from the

280 more susceptible muscle group TA (Figure 3C). Three genes from the PCDH cluster were

281 upregulated in FSHD2 cells consistent with the hypomethylation in this region (Figure 3C).

282 SMCHD1 expression is slightly lower in FSHD2 samples than control, which is surprising since

283 SMCHD1 mutations in these FSHD2 patients affect protein function, not expression (Figure 3C). 
We assessed global expression differences between FSHD2 and control combining all differentiation days. The most highly upregulated genes in FSHD2 include the FSHD-induced

287 genes previously identified (Figure 3B). The most significantly downregulated gene in FSHD2 is

288 FLVCR 1-AS1, a lncRNA, which can act as a sponge for miRNAs that inhibit proliferation

289 (Figure 3B) [43]. The genes for signaling molecules $F G F 7, F G F 10$ and TGFA are also down

290 regulated in FSHD2 compared to control (Figure 3B, Table S5). Using gene ontology analysis,

291 the differentially methylated regions were identified as being associated with genes involved in

292 histone modifications, including H3K4 methylation and H2A ubiquitination, and FGF signaling

293 (Figure 3D).

\section{LTR loci are highly expressed in muscle that is more susceptible to FSHD}

297 assess the extent of TE upregulation in our samples [24,44]. In FSHD2 cells, 580 repeat loci

298 comprised of 175 different TE types are upregulated in a time specific manner starting at day 3

299 of differentiation (Figure 4A, 4B). Of these 175 types, 79 are LTRs including significant

300 enrichment of ERVL-MaLR ( $\mathrm{p}=4.9 \mathrm{e}-92)$, ERVL ( $\mathrm{p}=2.6 \mathrm{e}-17)$ and ERV1 (p=2.7e-5) (Figure 4C).

301 Specific repeat types, such as THE1D, MLT2A1 and THE1C, which are regulated by DUX4, are

302 also significantly enriched and upregulated (Figure S5A) [24]. These loci are substantially

303 upregulated in FSHD2 TA and show only slight upregulation in quadricep at day 12 of

304 differentiation (Figure 4A). 

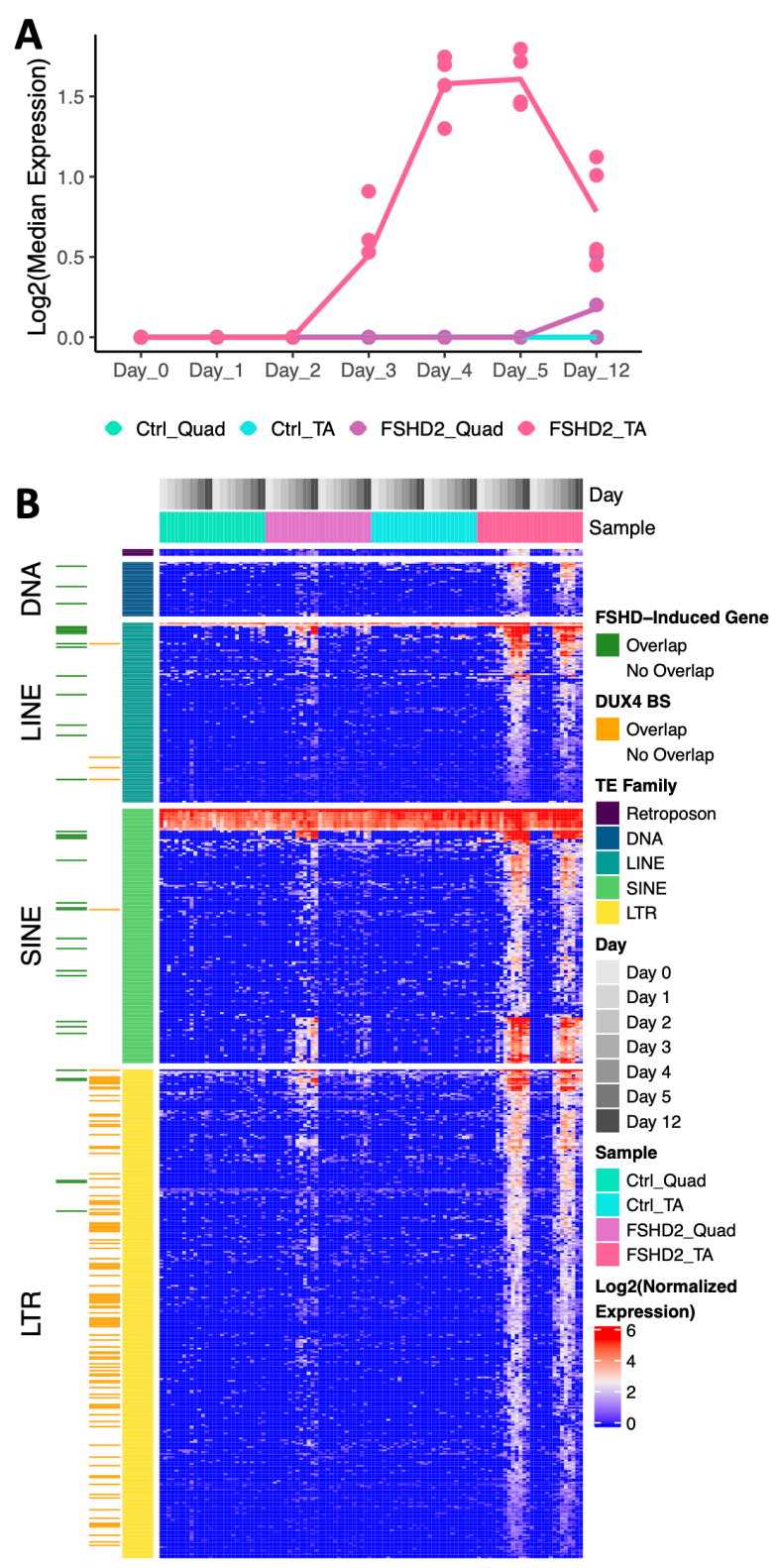
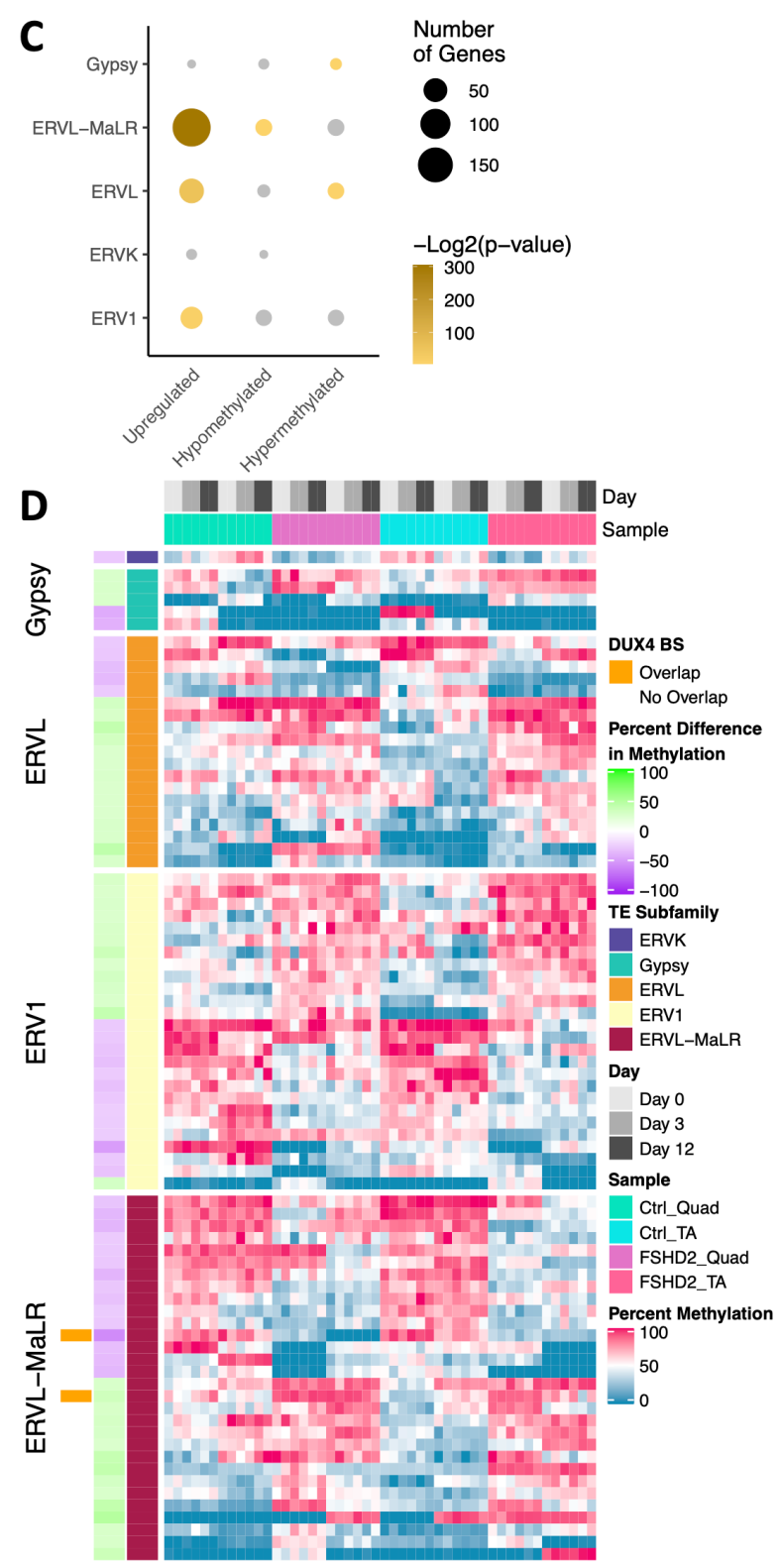

Figure 4: LTRs upregulated and hypomethylated in FSHD2 (A) Median expression for given

308 groups of 580 TEs with increased expression in FSHD2 during myogenesis. Dots represent

309 median expression for individual samples. Line represents the mean for the four samples in each

310 group. (B) Heatmap of expression of TEs with increased expression in FSHD2 during

311 myogenesis split by class of TE. Loci that overlap FSHD-induced genes are indicated in green on

312 the left. Loci which overlap known DUX4 binding sites are labelled in orange. (C) Enrichment 
313 of subclasses of LTRs in TEs upregulated in FSHD2 or differentially methylated in FSHD2.

314 Classes with p-value $>0.05$ are in grey. Size indicates number of loci in category. (D) Heatmap

315 of percent methylation for LTR loci with significant methylation differences between FSHD2

316 and control split by LTR subclass. Loci which overlap a known DUX4 binding site are indicated

317 in orange on the left.

These upregulated TEs significantly overlap $(p=2.3 e-70)$ DUX4 binding sites identified

321 by ChIP-seq (Figure 4B) [24]. Out of 104 loci that overlap DUX4 binding sites, 99 are LTRs

$322(\mathrm{p}=2.1 \mathrm{e}-28)$ including 79 ERVL-MaLRs $(\mathrm{p}=5.7 \mathrm{e}-24)$ (Figure 4B, 4C). To determine whether the

323 TEs are upregulated because they overlap FSHD-induced genes, we intersected the TE loci with

324 the FSHD-induced genes. Thirty-six TE loci overlap the FSHD-induced genes, only 6 of which

325 are LTRs (Figure 4B). A previous study found that LTRs bound by DUX4 could act as

326 alternative promoters for protein coding and lncRNAs [24]. We find that 20 out of the 79 LTRs

327 upregulated in FSHD2 overlap LTRs identified as alternative promoters, including 15 for protein

328 coding genes such as MLT1E1A-NT5C1B and 5 for lncRNAs (Table S6).

We summarized CpG sites over LTR loci to determine whether DUX4-regulated LTRs

331 are differentially methylated (Methods). Between FSHD2 and control, 45 LTRs are more highly

332 methylated (percent diff $>25 \%$, qvalue $<0.01$ ) in FSHD2, and 36 are more highly methylated in

333 control (Figure 4D). These loci were not in the set identified as upregulated in FSHD2. Loci

334 with more methylation in FSHD2 are enriched for ERVL $(p=0.01)$ and Gypsy $(p=0.04)$ elements

335 (Figure 4C). FSHD2 hypomethylated TEs are enriched for ERVL-MaLR ( $\mathrm{p}=0.03)$ which are 
336 enriched in the set of TEs upregulated in FSHD2 (Figure 4C). Additionally, two ERVL-MaLR

337 loci overlap DUX4 binding sites (Figure 4D).

339 Muscle group specific gene expression in FSHD

340 To identify transcriptional differences between muscle groups that may cause or result

341 from differential susceptibility to FSHD, we performed RNA-seq on FSHD1 patient matched

342 deltoid and bicep derived cell lines for days 0 and 5 of differentiation as well as from TA for day

3430 (Figure S1). We identified muscle specific expression patterns by performing pairwise

344 comparisons between the muscle groups at day 0 and taking the intersect of genes specific for

345 one muscle group (Methods). A total of 582 unique genes were specific to a muscle group with

346 either especially high or low expression for the group (Figure 5A). Bicep specifically expresses

$347 L H X 8$ which can regulate $S H H$ expression in development of various tissues in the upper body

348 (Figure 5B) [45]. Deltoid expresses HOXD3 which is involved in limb outgrowth (Figure 5B)

349 [46]. TA has higher expression of EYA1 which is involved in activating PAX3 and MYF5 in

350 limb muscles (Figure 5B) [5]. Quadricep has high expression of TLL1 which is involved in

351 dorsal ventral patterning and can indirectly regulate myostatin (Figure 5B) $[47,48]$. Interestingly,

352 we see the hindlimb specific TF TBX5 is high in the bicep, deltoid and quadricep but not the

353 tibialis anterior (Figure S6A). 
A
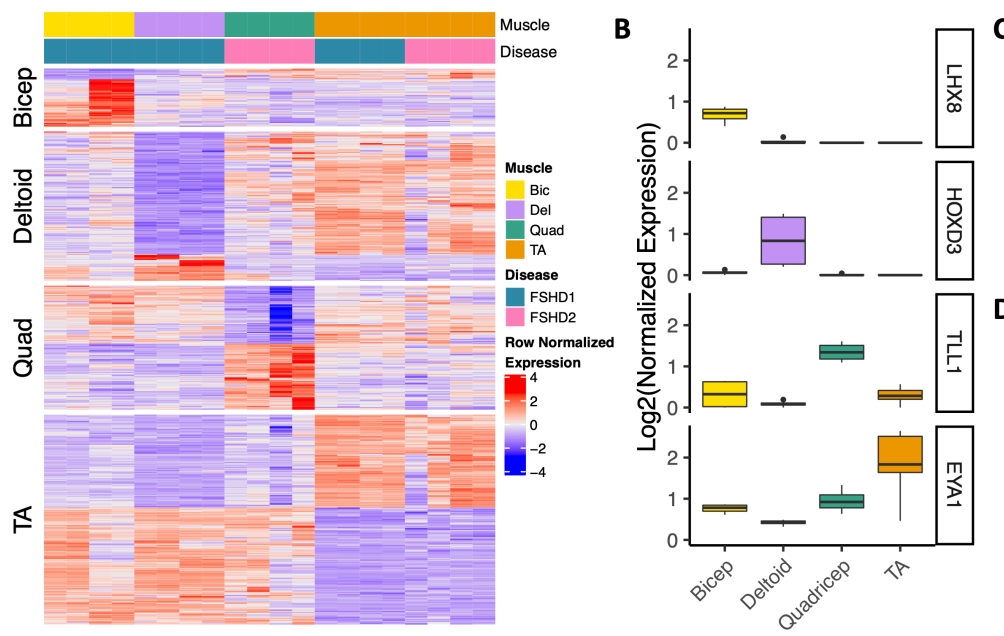

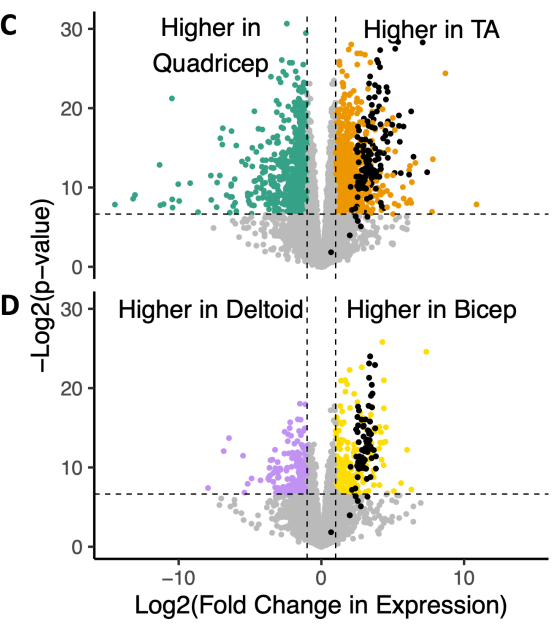

E

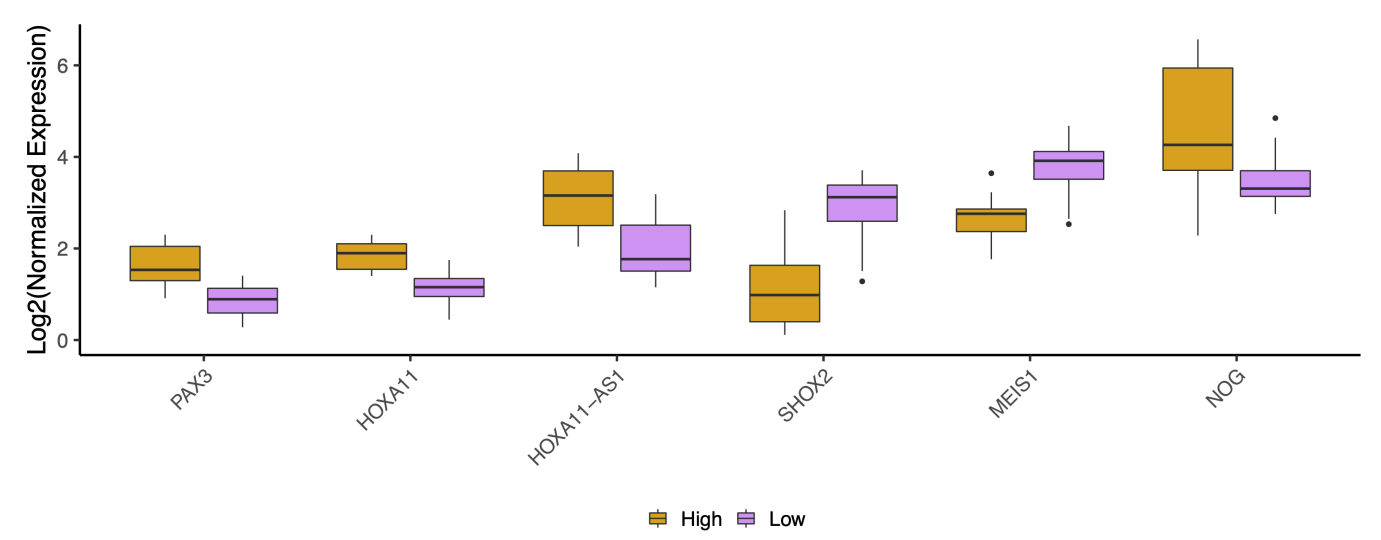

追 High 追 Low

Figure 5: Muscle group specific expression in muscles more and less susceptible to FSHD

(A) Heatmap of genes with muscle group specific expression. Top color bars indicate FSHD1 or FSHD2 and the muscle group of origin. (B) Boxplot of expression of four genes with muscle group specific expression. (C) Volcano plot of gene expression differences for FSHD2 TA versus FSHD2 quadricep at day 5 of differentiation. FSHD-induced genes are colored in black.

361 Genes with a p-value of $>0.01$ or an absolute $\log 2$ (fold change in expression) $<1$ are in grey.

362 Genes with higher expression in the TA or quadricep are in orange or green, respectively.

363 Vertical lines intersect y-axis at -1 and $1 \log 2$ (fold change). Horizontal line intersects y-axis at -

$364 \log 2(0.01)$. (D) Volcano plot of gene expression differences for FSHD1 bicep versus FSHD1 
366 value of $>0.01$ or an absolute $\log 2$ (fold change in expression) $<1$ are in grey. Genes with higher

367 expression in the bicep or deltoid are in yellow or purple, respectively. Vertical lines intersect y-

368 axis at -1 and $1 \log 2$ (fold change). Horizontal line intersects y-axis at $-\log 2(0.01)$. (E) Boxplot of

369 genes with differences in expression in muscle groups with high or low susceptibility to FSHD.

FSHD tends to affect upper musculature first, so we compared the two forelimb muscles

373 (bicep and deltoid) to the two hindlimb ones (quadricep and TA). Genes in the 5' end of the

374 HOXC cluster, including HOXC9 through 13 as well as HOXC-AS1, HOXC-AS5 and HOTAIR,

375 were all more highly expressed on average in the hindlimb than the forelimb (Figure S6B, S6C).

376 Genes in the 3' end of the HOXD cluster are more highly expressed in the forelimb (Figure

377 S6C).

We then compared expression between the commonly and less affected muscle groups in

380 the forelimb and hindlimb separately to investigate differences between muscle groups more

381 susceptible to FSHD. As noted previously, FSHD-induced genes are more highly expressed in

382 TA than quadricep, 71 out of 74 at day 5 of differentiation (Figure 5C). FSHD-induced genes are

383 also more highly expressed in bicep, which is more commonly affected, than deltoid (67 out of

384 74) (Figure 5D). Higher expression of DUX4 target genes has been shown previously to

385 correlate with active signs of disease [25]. Here we show that the FSHD gene signature is also

386 more highly expressed in more susceptible tissue. 
To find genes specific to commonly affected groups, we took the intersect of the genes differentially expressed in bicep compared to deltoid and TA compared to quadricep in the same

390 direction (Methods). This yields 28 genes more highly expressed in commonly affected groups, 391 and 27 higher in the less affected groups including 7 TFs total (Figure S6D, Table S7). The

392 myogenic precursor TF $P A X 3$ and $H O X A 11$ are more highly expressed in more susceptible

393 groups (Figure 5E). TFs more highly expressed in the less susceptible muscles include SHOX2

394 and MEIS1. MEIS1 and SHOX2 are expressed in the proximal region of the limb [8,9,49]. PAX3

395 marks myogenic precursor cells and may play a role in satellite cells postnatally [5]. HOXA11 is

396 expressed in the zeugopod in development $[8,10]$. We also see higher expression of genes

397 involved in WNT signaling including Noggin (Figure 5E, Figure S6C). Also higher in more

398 affected tissues is $H E Y L$ which is involved in NOTCH signaling, represses MYOD expression,

399 and is required for satellite cell proliferation in a model of hypertrophy (Figure S6C) [50,51]. In

400 conclusion, we identified DNA methylation and transcriptional differences between muscle

401 groups in cell lines derived from adult tissue.

\section{Discussion}

We identified developmental transcription factors with differences in DNA methylation

405 and expression in cells derived from adult tissue. By examining cells from FSHD patients, we

406 found differences between muscle groups that are related to disease, including PITX1,

407 emphasizing the consideration of sample origin in transcriptome studies in FSHD. Genes

408 induced specifically in FSHD are not differentially methylated, but genes regulated by SMCHD1

409 are hypomethylated and slightly overexpressed in SMCHD1 mutated FSHD2 patient cells. 
410 Importantly, we find a set of genes that possibly correlate with muscle group susceptibility to

411 FSHD.

412

The differential DNA methylation and gene expression between muscle groups supports

414 a potential role for developmental transcription factors in adult tissue. Previous work in mice

415 showed transcriptional and methylation differences between extraocular muscles (EOM) and TA

416 and that transplantation could alter the location-specific transcriptional profiles [15]. Notably,

417 EOM cells, which do not express HOX genes, upregulate TA specific HOX genes upon

418 transplantation supporting a role for environmental stimuli in controlling location-associated TFs

419 [15]. Notably, PITX2 expression in EOM cells was resistant to change after transplantation to the

420 TA, and PITX2 can increase the ability of satellite cells to regenerate $[4,15]$. We find substantial

421 methylation differences in PITX2 between TA and quadricep. The role of some of these other

422 TFs in adult tissue warrants further investigation.

The transcriptional differences that we observe between the bicep and TA compared with

425 the deltoid and quadricep may be the result of inherent differences between these muscle groups

426 or due specifically to disease. While the differences that we observed between TA and quadricep

427 could be due to individual differences, our bicep and deltoid samples were taken from the same

428 individuals. Comparison of these muscle groups from healthy individuals could identify whether

429 differences between the susceptible groups are due to inherent differences, active disease or

430 differences inherent to FSHD patients. The higher expression of FSHD-induced genes in the

431 more commonly affected muscle groups may be due to either active disease or inherent

432 differences between the groups. Indeed, FSHD-related gene expression has been shown to be 
433 higher in tissue with signs of active disease [25,26]. Differences in expression of homeobox

434 transcription factors between commonly and less affected groups suggests a possible link to the 435 governing of muscle groups affected.

438 little differential methylation compared to what we observe, which may be the result of increased 439 sample size or to the SMCHD1 mutations in the FSHD2 patients [52]. The enrichment in 440 hypomethylation of ERVL-MaLRs suggests a mechanism for their increased expression in

441 FSHD. The differentially methylated ERVL-MaLRs do not significantly overlap DUX4 binding

442 sites, which suggests that regulation of DNA methylation in those regions is independent of

443 DUX4 binding but could possibly be attributed to SMCHD1 mutations. In addition, we noted

444 differences in methylation related to FGF signaling and downregulation of two $F G F s$ and $T G F \alpha$

445 in FSHD2 cells. FGF10 is an important contributor to limb outgrowth but is largely unexplored

446 in FSHD. FGF1 and FGF2 were found in a biopsy of one patient with a severe phenotype [53].

447 We found NOG more highly expressed in more susceptible muscle groups in FSHD. Noggin

448 inhibits multiple BMPs including BMP4 [54]. BMP4 can activate FGF7 and FGF10 expression 449 [55].

The hypermethylation and lack of difference in the methylation of the FSHD-induced

452 gene promoters could indicate several possibilities. First, DUX4 most likely binds other

453 regulatory elements outside of these promoter regions, such as enhancers, such that highly

454 methylated promoters are not prohibitive to activation by DUX4. Second, DUX4 and/or other

455 binding partners may not be sensitive to DNA methylation. Indeed, homeobox transcription 
456 factors preferentially bind methylated DNA, and we find the methylated motif for DUXA

457 enriched at the promoters of FSHD-induced genes [35]. Second, complexes that are indirectly

458 called to methylated DNA, such as SIN3, regulate $D U X 4$ expression, and their targets are

459 affected in DUX4-affected cells [56-59]. DUX4 also upregulates the $M B D 3 L$ genes which are

460 methyl binding domain proteins, and MBD3L2 is known to bind methylated DNA [60,61].

461 Third, demethylated promoters may only be present in the subset of nuclei that activate the

462 DUX4 program. As affected FSHD nuclei make up a small percentage of total nuclei, differences

463 in those nuclei would only be observable with single nucleus methylation assays [23,57].

In summary, we identified transcriptional and DNA methylation differences between

466 muscle groups from adult tissue of healthy individuals and FSHD patients. We identified a set of

467 genes potentially linked to susceptibility or progression of affected muscle groups in FSHD,

468 including genes specifically upregulated in FSHD across myogenesis. Understanding the

469 genomic basis of susceptibility is important for identifying key mechanisms of FSHD

470 progression.

471

472 Methods

473

474 Human myoblast culture and differentiation

475 Human control, FSHD1 and FSHD2 myoblast cells from patient quadricep, tibialis

476 anterior, bicep and deltoid biopsies were grown as previously described [57]. Cells were cultured

477 on dishes coated with collagen in high glucose DMEM (Gibco) supplemented with 20\% FBS

478 (Omega Scientific, Inc.), 1\% Pen-Strep (Gibco), and 2\% Ultrasor G (Crescent Chemical Co.). 
Day 0 cells were kept at low confluency to prevent spontaneous differentiation. Upon reaching $80 \%$ confluence, differentiation was induced by using high glucose DMEM medium supplemented with 2\% FBS and ITS supplement (insulin $0.1 \%, 0.000067 \%$ sodium selenite, $0.055 \%$ transferrin; Invitrogen). Fresh differentiation medium was changed every 24 hours.

DNA methylation library preparation kit (69504, Qiagen). For both reps of day 0, day 3 and rep 1 of day 12 for Control-4, gDNA from two plates were pooled and concentrated using DNA Clean \& Concentrator-25 (D4033, Zymo) to obtain high enough input and concentration. All DNA methylation data was generated using the TruSeq Methyl Capture EPIC kit (FC-151-1003, Illumina) according to manufacturer's protocol [62]. Libraries were sequenced on the Illumina NextSeq500 with paired-end 75 bp reads

491 to a depth of 20 to 82 million reads.

\section{DNA methylation data processing}

496 (version 0.19.0) [63]. Sites were extracted from the bam file using

497 bismark_methylation_extractor with paired end and no overlap specified. To remove bias from

498 the ends of reads, 2 bp from the 5' end of read 2 and $1 \mathrm{bp}$ from the 3' end of read 1 were ignored

499 when extracting sites. All CpG sites were read into methylKit (version 1.16.0) [64]. Sites within

$500200 \mathrm{bp}$ were merged into one region using bumphunter (version 1.32.0) [65]. Methylation over

501 those regions was summarized using regionCounts and filtered for at least three CpG sites. 
502 Regions were then filtered for a coverage of at least 5. Regions were normalized using

503 normalizeCoverage, and only regions with coverage in all samples were kept. Differential

504 methylation was calculated using calculateDiffMeth with a chi-squared test with basic

505 overdispersion correction. Differentially methylated regions (DMRs) were defined with a q-

506 value $<0.01$ and a percent difference in methylation of at least $25 \%$ for TA versus quadricep and

507 FSHD2 versus control for TE loci but 10\% for FSHD2 versus control.

Regions were associated with genes and gene ontology using rGREAT (version 1.20.0)

510 [32] with submitGreatJob with hg38 specified. GO term plots include the top 10 terms with

511 greater than 10 genes associated with the background set for the term and a Benjamini-Hochberg

512 corrected p-value of less than 0.05. Regions were overlapped with SMCHD1 regulated regions

513 and DUX4 binding sites from [24] using findOverlaps from GenomicRanges (version 1.40.0)

$514[66]$

515

For the promoter analysis, $\mathrm{CpG}$ sites were summarized for $1.5 \mathrm{~kb}$ upstream and $0.5 \mathrm{~kb}$

517 downstream of the TSS using regionCounts and filtered for at least $1 \mathrm{CpG}$ site. Promoters were

518 filtered for a coverage of at least 5 then normalized. Promoters detected in all samples were kept

519 for further analysis as described.

521 Motif analysis

522 Motif enrichment was performed on DMRs extended $24 \mathrm{bp}$ in both directions since the

523 regions end on $\mathrm{CpG}$ sites. Enrichment was performed using AME (meme, version 5.2.0) [67] on

524 JASPAR 2020 core redundant motifs from human only [68]. Association of TFs with GO terms 
525 for figure $1 \mathrm{C}$ was done by associating the TFs with the following GO terms: limb

526 morphogenesis, embryonic limb morphogenesis, embryonic forelimb morphogenesis, limb

527 development, limb bud formation for limb; anterior/posterior pattern specification,

$528 \mathrm{proximal} /$ distal pattern formation, dorsal/ventral pattern formation, dorsal/ventral pattern

529 specification for axis patterning; skeletal muscle tissue development, skeletal system

530 development, negative regulation of myoblast differentiation, muscle organ development,

531 skeletal muscle cell differentiation, skeletal muscle tissue regeneration, myoblast development,

532 and positive regulation of myoblast proliferation for muscle. Upset plots were created using

533 UpsetR (version 1.4.0) [69].

535 TE methylation analysis

For the TE analysis, CpG sites were summarized over LTR loci from repeatmasker taken

537 from UCSC and filtered for at least $1 \mathrm{CpG}$ site. Loci were filtered for a coverage of at least 5

538 then normalized. Loci detected in all samples were kept for further analysis as described.

540 RNA sequencing library preparation

Total RNA was collected using the RNEasy kit (74106, Qiagen). RNA was converted to

542 cDNA using the Smart-Seq2 protocol [70]. Libraries were constructed with the Nextera DNA

543 Library Prep Kit (Illumina) for days 0 to 5 for Control-3, Control-4, FSHD2-2 and FSHD2-3.

544 The Nextera DNA Flex Library Prep Kit (Illumina) was used for all other RNA-seq samples.

545 Libraries were sequenced on the Illumina NextSeq500 with paired-end 43 bp reads to a depth of

5465 to 40 million reads. Data for days 0 through 5 for Control-1, Control-2, Control-3, Control-4,

547 FSHD2-1 and FSHD2-2 were obtained from GEO (accession number GSE143493). 
RNA sequencing data processing using defaults except with a maximum of 10 mismatches per pair, a ratio of mismatches to read

552 length of 0.07 , and a maximum of 10 multiple alignments. Quantitation was performed using

553 RSEM (version 1.2.31) [72] with defaults with gene annotations for protein coding genes and

554 lncRNAs from GENCODE v28. Counts were batch corrected for the two different library prep 555 methods using ComBat-seq from sva (version 3.36.0) [73]. Genes were filtered for 10 counts in 556 all samples of at least one condition (same FSHD status, muscle group and differentiation day)

557 using filterByExpr from edgeR (version 3.30.3) [74]. Normalization for days 0 to 5 and 12 for 558 control and FSHD2 for figures 1 through 3 was performed separately from days 0 and 5 for 559 FSHD2 and FSHD1 for figure 5. TMM normalized counts from edgeR were used for differential 560 expression analysis in edgeR and clustering genes into similar expression profiles using 561 maSigPro (version 1.60.0) [75]. TMM normalized counts were TPM normalized for plotting 562 using effective gene lengths for each sample calculated by RSEM. Heatmaps were created using 563 ComplexHeatmap (version 2.4.3) [76]. Median expression of gene groups for each sample was 564 calculated by taking the median TPM normalized TMM values for all genes, and lines represent 565 the mean of the median values for the given samples. Muscle specific genes were identified by 566 performing pairwise comparisons between muscle groups (i.e. TA vs quadricep, TA vs deltoid, 567 TA vs bicep) as described with edgeR and taking the intersect of all genes specific to the same 568 muscle group in all comparisons (i.e. up in all TA comparisons). Genes with higher expression in 569 more or less susceptible tissue were identified by intersecting the genes higher in TA than 
570 quadricep and higher in bicep than deltoid and vice versa. The list of transcription factors was

571 taken from AnimalTFDB (version 3.0) [77].

572

$573 \quad$ TE mapping and processing

574 To map to TEs, fastqs were aligned as described above to the full GENCODE v28

575 annotation with a maximum of 100 multiple alignments and maximum of 100 loci anchors.

576 Reads mapping to TE loci from repeatmasker from UCSC were estimated using featureCounts

577 (subread version 2.0.1) [78] with fractional counts for multimapped reads for paired end reads.

578 Genes were filtered for 2 counts in all samples of at least one condition (same FSHD status,

579 muscle group and differentiation day) using filterByExpr from edgeR (version 3.30.3) [74].

580 Counts were normalized as described above. Loci were clustered into similar expression profiles

581 as described above. Loci were overlapped with FSHD-induced genes (TSS to TES) or DUX4

582 binding sites from [24] using findOverlaps from GenomicRanges (version 1.40.0) [66]. Fisher's

583 exact test (stats version 4.0.2) was used for enrichment of TE classes and DUX4 binding site

584 overlaps with the "greater" alternative hypothesis.

585

586 Processing of publicly available data

$587 \quad$ Fastqs for the biopsy RNA-seq data from $[21,25,79,80]$ were obtained from GEO with

588 the accessions in table 1. Fastqs were processed in the same way as bulk RNA-seq. Counts were

589 normalized as described above but with no batch correction.

590

591

Table 1: GEO Accession numbers for fastqs from previous studies

\begin{tabular}{|l|l|l|l|}
\hline Disease & Muscle & SRA & Reference \\
\hline Ctrl & Quad & SRR7293780 & {$[25]$} \\
\hline Ctrl & Quad & SRR7293781 & {$[25]$} \\
\hline Ctrl & Quad & SRR7293782 & {$[25]$} \\
\hline
\end{tabular}




\begin{tabular}{|c|c|c|c|}
\hline $\mathrm{Ctrl}$ & Quad & SRR7293783 & {$[25]$} \\
\hline $\mathrm{Ctrl}$ & Quad & SRR7293784 & {$[25]$} \\
\hline Ctrl & Quad & SRR7293785 & {$[25]$} \\
\hline $\mathrm{Ctrl}$ & Quad & SRR7293793 & {$[25]$} \\
\hline Ctrl & Quad & SRR7293794 & {$[25]$} \\
\hline Ctrl & Quad & SRR7293795 & {$[25]$} \\
\hline FSHD & Quad & SRR7293761 & {$[25]$} \\
\hline FSHD & Quad & SRR7293769 & {$[25]$} \\
\hline FSHD & Quad & SRR7293774 & {$[25]$} \\
\hline FSHD & Quad & SRR7293786 & {$[25]$} \\
\hline FSHD & Quad & SRR7293788 & {$[25]$} \\
\hline FSHD & Quad & SRR7293801 & {$[25]$} \\
\hline FSHD & TA & SRR7293759 & {$[25]$} \\
\hline FSHD & TA & SRR7293763 & {$[25]$} \\
\hline FSHD & TA & SRR7293766 & {$[25]$} \\
\hline FSHD & TA & SRR7293767 & {$[25]$} \\
\hline FSHD & TA & SRR7293770 & {$[25]$} \\
\hline FSHD & TA & SRR7293771 & {$[25]$} \\
\hline FSHD & TA & SRR7293787 & {$[25]$} \\
\hline FSHD & TA & SRR7293790 & {$[25]$} \\
\hline FSHD & $\mathrm{TA}$ & SRR7293791 & {$[25]$} \\
\hline FSHD & TA & SRR7293792 & {$[25]$} \\
\hline FSHD & TA & SRR7293796 & {$[25]$} \\
\hline FSHD & TA & SRR7293799 & {$[25]$} \\
\hline FSHD & TA & SRR7293800 & [25] \\
\hline MB135_HDUX4CA_nodox_rep1 & NA & SRR4019004 & [79] \\
\hline MB135_HDUX4CA_WITHdox_rep1 & NA & SRR4019005 & {$[79]$} \\
\hline MB135_HDUX4CA_nodox_rep2 & NA & SRR4019006 & [79] \\
\hline MB135_HDUX4CA_WITHdox_rep2 & NA & SRR4019007 & [79] \\
\hline MB135_HDUX4CA_nodox_rep3 & NA & SRR4019008 & [79] \\
\hline MB135_HDUX4CA_WITHdox_rep3 & NA & SRR4019009 & [79] \\
\hline FSHD_1_1_neg & NA & SRR2020583 & {$[80]$} \\
\hline FSHD_1_2_neg & NA & SRR2020584 & {$[80]$} \\
\hline FSHD_2_2_BFP & NA & SRR2020585 & {$[80]$} \\
\hline FSHD_2_3_BFP & NA & SRR2020586 & {$[80]$} \\
\hline FSHD_1_3_neg & NA & SRR2020587 & {$[80]$} \\
\hline FSHD_1_1_BFP & NA & SRR2020588 & {$[80]$} \\
\hline FSHD_1_2_BFP & NA & SRR2020589 & {$[80]$} \\
\hline FSHD_1_3_BFP & NA & SRR2020590 & {$[80]$} \\
\hline
\end{tabular}




\begin{tabular}{|l|l|l|l|}
\hline FSHD_2_1_neg & NA & SRR2020591 & {$[80]$} \\
\hline FSHD_2_2_neg & NA & SRR2020592 & {$[80]$} \\
\hline FSHD_2_3_neg & NA & SRR2020593 & {$[80]$} \\
\hline FSHD_2_1_BFP & NA & SRR2020594 & {$[80]$} \\
\hline Control_20_Mt & NA & SRR1398556 & {$[21]$} \\
\hline Contro1_21_Mb & NA & SRR1398557 & {$[21]$} \\
\hline Contro1_21_Mt & NA & SRR1398558 & {$[21]$} \\
\hline Control_22_Mb & NA & SRR1398559 & {$[21]$} \\
\hline Control_22_Mt & NA & SRR1398560 & {$[21]$} \\
\hline FSHD2_12_Mt & NA & SRR1398561 & {$[21]$} \\
\hline FSHD2_14_Mb & NA & SRR1398562 & {$[21]$} \\
\hline FSHD2_14_Mt & NA & SRR1398563 & {$[21]$} \\
\hline FSHD2_20_Mb & NA & SRR1398564 & {$[21]$} \\
\hline FSHD2_20_Mt & NA & SRR1398565 & {$[21]$} \\
\hline FSHD1_4_Mb & NA & SRR1398566 & {$[21]$} \\
\hline FSHD1_4_Mt & NA & SRR1398567 & {$[21]$} \\
\hline FSHD1_6_Mb & NA & SRR1398568 & {$[21]$} \\
\hline FSHD1_6_Mt & NA & SRR1398569 & {$[21]$} \\
\hline
\end{tabular}

\section{Data Availability}

594 Data generated for this manuscript are available through GEO (GSE174370) or through dbGap 595 (accession number TBD).

\section{Funding}

598 This work was funded in part from grant AR071287 from NIAMS to AM and KY.

600 Competing Interests

601 The authors declare no competing interests.

602

\section{Authors' contributions}

604 KW - Conceptualization, Data curation, Formal analysis, Investigation, Writing - original draft,

605 Writing - review \& editing 
606 XK - Investigation, Resources

$607 \quad \mathrm{NVN}-$ Investigation

608 CM - Resources

609 RT - Resources

610 KY - Funding acquisition, Project administration, Resources, Supervision, Writing - review \&

611 editing

612 AM - Funding acquisition, Project administration, Resources, Supervision, Writing - review \&

613 editing

614

615 Acknowledgements

616 We would like to thank the NIH UMMS Senator Paul D. Wellstone Muscular Dystrophy

617 Cooperative Research Center for FSHD Research for providing the bicep and deltoid cell lines. 


\section{References}

621 1. Science Reference Section L of C. What is the strongest muscle in the human body? [Internet]. 2019. Available from: https://www.loc.gov/everyday-mysteries/item/what-is-

2. Chal J, Pourquié O. Making muscle: Skeletal myogenesis in vivo and in vitro. Dev. 2017;144(12):2104-22.

3. Edgerton VR, Smith JL, Simpson DR. Muscle fibre type populations of human leg muscles. Histochem J. 1975;7(3):259-66.

4. Hernandez-Torres F, Rodríguez-Outeiriño L, Franco D, Aranega AE. Pitx2 in embryonic and adult myogenesis. Vol. 5, Frontiers in Cell and Developmental Biology. Frontiers Media S.A.; 2017. p. 46.

5. Buckingham M, Rigby PWJ. Gene Regulatory Networks and Transcriptional Mechanisms that Control Myogenesis. Vol. 28, Developmental Cell. 2014. p. 225-38.

6. L'Honoré A, Ouimette JF, Lavertu-Jolin M, Drouin J. Pitx2 defines alternate pathways acting through MyoD during limb and somitic myogenesis. Development. 2010;137(22):3847-56.

7. Braun T, Gautel M. Transcriptional mechanisms regulating skeletal muscle differentiation, growth and homeostasis. Nat Rev Mol Cell Biol. 2011;12(6):349-61.

8. McQueen C, Towers M. Establishing the pattern of the vertebrate limb. Development. 2020 Sep 1;147(17):dev177956.

9. Ye W, Song Y, Huang Z, Osterwalder M, Ljubojevic A, Xu J, et al. A unique stylopod patterning mechanism by shox2-controlled osteogenesis. Dev. 2016;143(14):2548-60.

10. Delgado I, Torres M. Gradients, waves and timers, an overview of limb patterning models. Semin Cell Dev Biol. 2016;49:109-15.

11. Ahn K, Mishina Y, Hanks MC, Behringer RR, Bryan Crenshaw E. BMPR-IA signaling is required for the formation of the apical ectodermal ridge and dorsal-ventral patterning of the limb. Development. 2001;128(22):4449-61.

12. Kang PB, Kho AT, Sanoudou D, Haslett JN, Dow CP, Han M, et al. Variations in gene expression among different types of human skeletal muscle. Muscle Nerve. 2005 Oct 1;32(4):483-91.

13. Porter JD, Merriam AP, Leahy P, Gong B, Feuerman J, Cheng G, et al. Temporal gene expression profiling of dystrophin-deficient ( $\mathrm{mdx}$ ) mouse diaphragm identifies conserved and muscle group-specific mechanisms in the pathogenesis of muscular dystrophy. Hum Mol Genet. 2004;13(3):257-69.

14. Terry EE, Zhang X, Hoffmann C, Hughes LD, Lewis SA, Li J, et al. Transcriptional profiling reveals extraordinary diversity among skeletal muscle tissues. Elife. 2018 May $29 ; 7$.

15. Evano B, Gill D, Hernando-Herraez I, Comai G, Stubbs TM, Commere PH, et al. Transcriptome and epigenome diversity and plasticity of muscle stem cells following transplantation. PLoS Genet. 2020 Oct 30;16(10):e1009022.

16. Ciciliot S, Rossi AC, Dyar KA, Blaauw B, Schiaffino S. Muscle type and fiber type specificity in muscle wasting. Int J Biochem Cell Biol. 2013;45(10):2191-9.

17. Wagner KR. Facioscapulohumeral Muscular Dystrophies. Muscle Neuromuscul Junction Disord. 2019;25(6):1662-81.

18. Olsen DB, Gideon P, Jeppesen TD, Vissing J. Leg muscle involvement in facioscapulohumeral muscular dystrophy assessed by MRI. J Neurol. 2006;253(11):1437- 
666

41.

19. Statland JM, Tawil R. Facio-scapulo-humeral muscular dystrophy. Indian J Pediatr. 2008;34(5):186-8.

20. Lu J, Yao Z, Yang Y, Zhang C, Zhang J, Zhang Y. Management strategies in facioscapulohumeral muscular dystrophy. Intractable Rare Dis Res. 2019;8(1):9-13.

21. Yao Z, Snider L, Balog J, Lemmers RJLF, Van Der Maarel SM, Tawil R, et al. DUX4induced gene expression is the major molecular signature in FSHD skeletal muscle. Hum Mol Genet. 2014 Oct 15;23(20):5342-52.

22. Geng LN, Yao Z, Snider L, Fong AP, Cech JN, Young JM, et al. DUX4 Activates Germline Genes, Retroelements, and Immune Mediators: Implications for Facioscapulohumeral Dystrophy. Dev Cell. 2012 Jan 17;22(1):38-51.

23. Tassin A, Laoudj-Chenivesse D, Vanderplanck C, Barro M, Charron S, Ansseau E, et al. DUX4 expression in FSHD muscle cells: How could such a rare protein cause a myopathy? J Cell Mol Med. 2013 Jan;17(1):76-89.

24. Young JM, Whiddon JL, Yao Z, Kasinathan B, Snider L, Geng LN, et al. DUX4 Binding to Retroelements Creates Promoters That Are Active in FSHD Muscle and Testis. PLoS Genet. 2013 Nov;9(11).

25. Wang LH, Friedman SD, Shaw D, Snider L, Wong CJ, Budech CB, et al. MRI-informed muscle biopsies correlate MRI with pathology and DUX4 target gene expression in FSHD. Hum Mol Genet. 2019 Feb 1;28(3):476-86.

26. Wong CJ, Wang LH, Friedman SD, Shaw D, Campbell AE, Budech CB, et al. Longitudinal measures of RNA expression and disease activity in FSHD muscle biopsies. Hum Mol Genet. 2020;29(6):1030-44.

27. Rahimov F, King OD, Leung DG, Bibat GM, Emerson CP, Kunkel LM, et al. Transcriptional profiling in facioscapulohumeral muscular dystrophy to identify candidate biomarkers. Proc Natl Acad Sci. 2012 Sep 18;109(40):16234-9.

28. Daubas P, Duval N, Bajard L, Vives FL, Robert B, Mankoo BS, et al. Fine-tuning the onset of myogenesis by homeobox proteins that interact with the Myf5 limb enhancer. Biol Open. 2015;4(12):1614-24.

29. Ouimette JF, Jolin ML, L'honoré A, Gifuni A, Drouin J. Divergent transcriptional activities determine limb identity. Nat Commun. 2010 Jul 13;1(4):1-9.

30. Draut H, Liebenstein T, Begemann G. New insights into the control of cell fate choices and differentiation by retinoic acid in cranial, axial and caudal structures. Biomolecules. 2019;9(12).

31. Sturgeon K, Kaneko T, Biemann M, Gauthier A, Chawengsaksophak K, Cordes SP. Cdx1 refines positional identity of the vertebrate hindbrain by directly repressing $\mathrm{Mafb}$ expression. Development. 2011;138(1):65-74.

32. Mclean CY, Bristor D, Hiller M, Clarke SL, Schaar BT, Lowe CB, et al. GREAT improves functional interpretation of cis-regulatory regions HHS Public Access Author manuscript. Nat Biotechnol. 2010;28(5):495-501.

33. Koshiba-Takeuchi K, Takeuchi JK, Arruda EP, Kathiriya IS, Mo R, Hui CC, et al. Cooperative and antagonistic interactions between Sall4 and Tbx5 pattern the mouse limb and heart. Nat Genet. 2006;38(2):175-83.

34. Li D, Sakuma R, Vakili NA, Mo R, Puviindran V, Deimling S, et al. Formation of proximal and anterior limb skeleton requires early function of $\operatorname{Irx} 3$ and $\operatorname{Irx} 5$ and is negatively regulated by shh signaling. Dev Cell. 2014;29(2):233-40. 
35. Yin Y, Morgunova E, Jolma A, Kaasinen E, Sahu B, Khund-Sayeed S, et al. Impact of cytosine methylation on DNA binding specificities of human transcription factors. Science (80- ). 2017 May 5;356(6337).

36. Katayama S, Ranga V, Jouhilahti E-M, Airenne TT, Johnson MS, Mukherjee K, et al. Phylogenetic and mutational analyses of human LEUTX, a homeobox gene implicated in embryogenesis. Sci Rep. 2018 Dec 27;8(1):17421.

37. Himeda CL, Jones TI, Jones PL. Facioscapulohumeral muscular dystrophy as a model for epigenetic regulation and disease. Antioxid Redox Signal. 2015 Jun 1;22(16):1463-82.

38. Greco A, Goossens R, van Engelen B, van der Maarel SM. Consequences of epigenetic derepression in facioscapulohumeral muscular dystrophy. Clin Genet. 2020;97(6):799814.

39. de Greef JC, Lemmers RJLF, van Engelen BGM, Sacconi S, Venance SL, Frants RR, et al. Common epigenetic changes of D4Z4 in contraction-dependent and contractionindependent FSHD. Hum Mutat. 2009 Oct;30(10):1449-59.

40. Lemmers RJLF, Tawil R, Petek LM, Balog J, Block GJ, Santen GWE, et al. Digenic inheritance of an SMCHD1 mutation and an FSHD-permissive D4Z4 allele causes facioscapulohumeral muscular dystrophy type 2. Nat Genet. 2012 Dec;44(12):1370-4.

41. Mason AG, Slieker RC, Balog J, Lemmers RJLF, Wong CJ, Yao Z, et al. SMCHD1 regulates a limited set of gene clusters on autosomal chromosomes. Skelet Muscle. 2017 Jun 6;7(1).

42. Jansz N, Chen K, Murphy JM, Blewitt ME. The Epigenetic Regulator SMCHD1 in Development and Disease. Vol. 33, Trends in Genetics. Elsevier Ltd; 2017. p. 233-43.

43. Pan Z, Ding J, Yang Z, Li H, Ding H, Chen Q. LncRNA FLVCR1-AS1 promotes proliferation, migration and activates Wnt/ $\beta$-catenin pathway through miR-3813p/CTNNB1 axis in breast cancer. Cancer Cell Int. 2020;20(1):1-12.

44. Hendrickson PG, Doráis JA, Grow EJ, Whiddon JL, Lim JW, Wike CL, et al. Conserved roles of mouse DUX and human DUX4 in activating cleavage-stage genes and MERVL/HERVL retrotransposons. Nat Genet. 2017 Jun 1;49(6):925-34.

45. Zhou C, Yang G, Chen M, He L, Xiang L, Ricupero C, et al. Lhx6 and Lhx8: Cell fate regulators and beyond. FASEB J. 2015;29(10):4083-91.

46. Tarchini B, Duboule D. Control of Hoxd genes' collinearity during early limb development. Dev Cell. 2006;10(1):93-103.

47. Hopkins DR, Keles S, Greenspan DS. The Bone Morphogenetic Protein 1/Tolloid-like Metalloproteinases. Bone. 2007;26(7):508-23.

48. Allen DL, Greyback BJ, Hanson AM, Cleary AS, Lindsay SF. Skeletal muscle expression of bone morphogenetic protein-1 and tolloid-like-1 extracellular proteases in different fiber types and in response to unloading, food deprivation and differentiation. J Physiol Sci. 2010;60(5):343-52.

49. Petit F, Sears KE, Ahituv N. Limb development: A paradigm of gene regulation. Nat Rev Genet. 2017;18(4):245-58.

50. Fukuda S, Kaneshige A, Kaji T, Noguchi YT, Takemoto Y, Zhang L, et al. Sustained expression of HeyL is critical for the proliferation of muscle stem cells in overloaded muscle. Elife. 2019;8:1-21.

51. Noguchi YT, Nakamura M, Hino N, Nogami J, Tsuji S, Sato T, et al. Cell-autonomous and redundant roles of Heyl and HeyL in muscle stem cells: HeyL requires HeS1 to bind diverse DNA sites. Dev. 2019;146(4):1-12. 
52. Tsumagari K, Baribault C, Terragni J, Varley KE, Gertz J, Pradhan S, et al. Early de novo DNA methylation and prolonged demethylation in the muscle lineage. Epigenetics. 2013;8(3):317-32.

53. Saito A, Higuchi I, Nakagawa M, Saito M, Uchida Y, Inose M, et al. An overexpression of fibroblast growth factor (FGF) and FGF receptor 4 in a severe clinical phenotype of facioscapulohumeral muscular dystrophy. Muscle Nerve. 2000;4(23):490-7.

54. Zimmerman LB, De Jesús-Escobar JM, Harland RM. The Spemann organizer signal noggin binds and inactivates bone morphogenetic protein 4. Cell. 1996;86(4):599-606.

55. Gozo MC, Aspuria PJ, Cheon DJ, Walts AE, Berel D, Miura N, et al. Foxc2 induces Wnt4 and Bmp4 expression during muscle regeneration and osteogenesis. Cell Death Differ. 2013;20(8):1031-42.

56. van den Heuvel A, Mahfouz A, Kloet SL, Balog J, van Engelen BGM, Tawil R, et al. Single-cell RNA sequencing in facioscapulohumeral muscular dystrophy disease etiology and development. Hum Mol Genet. 2018 Nov 16;

57. Williams K, Jiang S, Kong X, Zeng W, Nguyen NV, Ma X, et al. Single-nucleus RNA-seq identifies divergent populations of FSHD2 myotube nuclei. PLoS Genet. 2020;16(5):1-26.

58. Cramer JM, Pohlmann D, Gomez F, Mark L, Kornegay B, Hall C, et al. Methylation specific targeting of a chromatin remodeling complex from sponges to humans. Sci Rep. 2017;7:1-15.

59. Grzenda A, Lomberk G, Zhang J-S, Urrutia R. Sin3: Master Scaffold and Transcriptional Corepressor Adrienne. Biochim Biophys Acta. 2009;0(1789):443-50.

60. Peng L, Li Y, Xi Y, Li W, Li J, Lv R, et al. MBD3L2 promotes Tet2 enzymatic activity for mediating 5-methylcytosine oxidation. J Cell Sci. 2016 Mar 1;129(5):1059-71.

61. Campbell AE, Shadle SC, Jagannathan S, Lim J-W, Resnick R, Tawil R, et al. NuRD and CAF-1-mediated silencing of the D4Z4 array is modulated by DUX4-induced MBD3L proteins. Elife. 2018 Mar 13;7.

62. Illumina. TruSeq Methyl Capture EPIC Library Prep Kit. 2017.

63. Krueger F, Andrews SR. Bismark: a flexible aligner and methylation caller for BisulfiteSeq applications. Bioinformatics. 2011 Jun 1;27(11):1571-2.

64. Akalin A, Kormaksson M, Li S, Garrett-Bakelman FE, Figueroa ME, Melnick A, et al. methylKit: a comprehensive R package for the analysis of genome-wide DNA methylation profiles. Genome Biol. 2012 Oct 3;13(10):R87.

65. Jaffe AE, Murakami P, Lee H, Leek JT, Fallin MD, Feinberg AP, et al. Bump hunting to identify differentially methylated regions in epigenetic epidemiology studies. Int $\mathrm{J}$ Epidemiol. 2012;41(1):200-9.

66. Lawrence M, Huber W, Pagès H, Aboyoun P, Carlson M, Gentleman R, et al. Software for Computing and Annotating Genomic Ranges. PLoS Comput Biol. 2013;9(8):1-10.

67. McLeay RC, Bailey TL. Motif Enrichment Analysis: A unified framework and an evaluation on ChIP data. BMC Bioinformatics. 2010;11.

68. Fornes O, Castro-Mondragon JA, Khan A, Van Der Lee R, Zhang X, Richmond PA, et al. JASPAR 2020: Update of the open-Access database of transcription factor binding profiles. Nucleic Acids Res. 2020;48(D1):D87-92.

69. Conway JR, Lex A, Gehlenborg N. UpSetR: An R package for the visualization of intersecting sets and their properties. Bioinformatics. 2017;33(18):2938-40.

70. Picelli S, Faridani OR, Björklund ÅK, Winberg G, Sagasser S, Sandberg R. Full-length RNA-seq from single cells using Smart-seq2. Nat Protoc. 2014;9(1):171-81. 
804 71. Dobin A, Davis CA, Schlesinger F, Drenkow J, Zaleski C, Jha S, et al. STAR: ultrafast universal RNA-seq aligner. Bioinformatics. 2013 Jan 1;29(1):15-21.

72. Li B, Dewey CN. RSEM: accurate transcript quantification from RNA-Seq data with or without a reference genome. BMC Bioinformatics. 2011 Dec 4;12(1):323.

808 73. Zhang Y, Parmigiani G, Johnson WE. ComBat-seq: batch effect adjustment for RNA-seq count data. NAR Genomics Bioinforma. 2020;2(3):1-10.

74. Robinson MD, McCarthy DJ, Smyth GK. edgeR: a Bioconductor package for differential expression analysis of digital gene expression data. Bioinformatics. 2010 Jan 1;26(1):139_40.

75. Conesa A, Nueda M. maSigPro: Significant Gene Expression Profile Differences in Time Course Gene Expression Data. 2017.

76. Gu Z, Eils R, Schlesner M. Complex heatmaps reveal patterns and correlations in multidimensional genomic data. Bioinformatics. 2016 Sep 15;32(18):2847-9.

77. Hu H, Miao Y-R, Jia L-H, Yu Q-Y, Zhang Q, Guo A-Y. AnimalTFDB 3.0: a comprehensive resource for annotation and prediction of animal transcription factors. Nucleic Acids Res. 2019 Jan 8;47(D1):D33-8.

78. Liao Y, Smyth GK, Shi W. FeatureCounts: An efficient general purpose program for assigning sequence reads to genomic features. Bioinformatics. 2014;30(7):923-30.

79. Jagannathan S, Shadle SC, Resnick R, Snider L, Tawil RN, van der Maarel SM, et al. Model systems of DUX4 expression recapitulate the transcriptional profile of FSHD cells. Hum Mol Genet. 2016 Aug 17;ddw271.

80. Rickard AM, Petek LM, Miller DG. Endogenous DUX4 expression in FSHD myotubes is sufficient to cause cell death and disrupts RNA splicing and cell migration pathways. Hum Mol Genet. 2015 Jun 5;24(20):5901-14. 
A
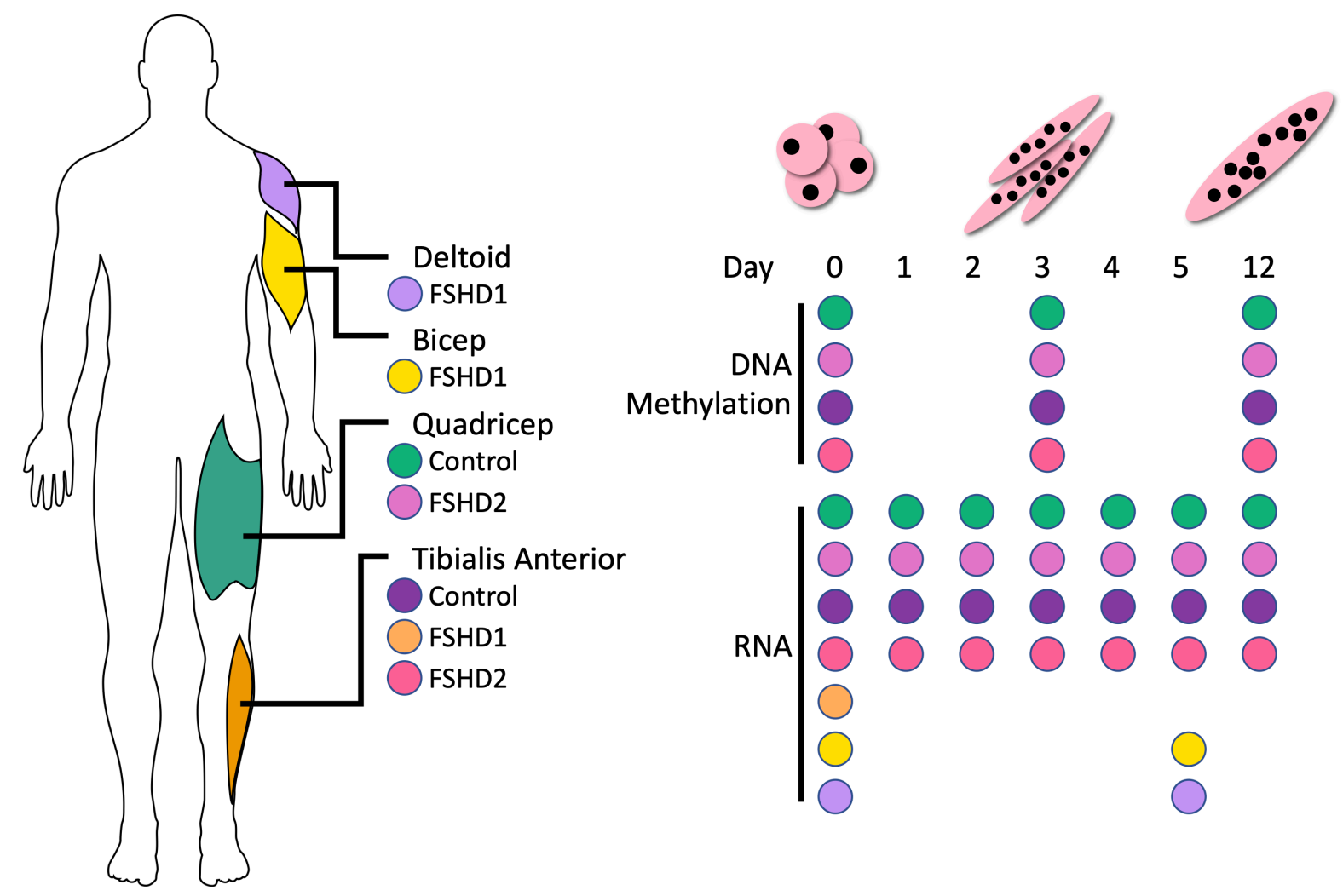

833 Figure S1: Experiment overview (A) Overview of samples used for bisulfite and RNA

834 sequencing. 


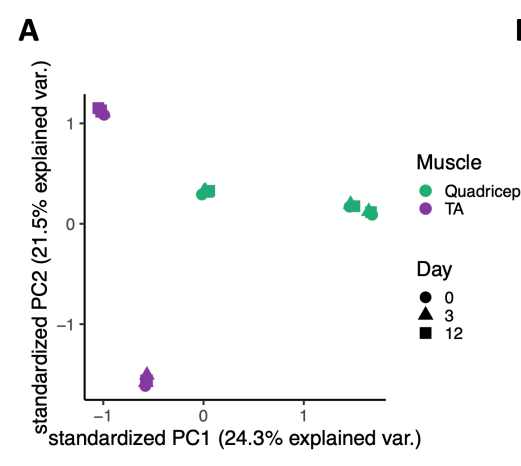

B
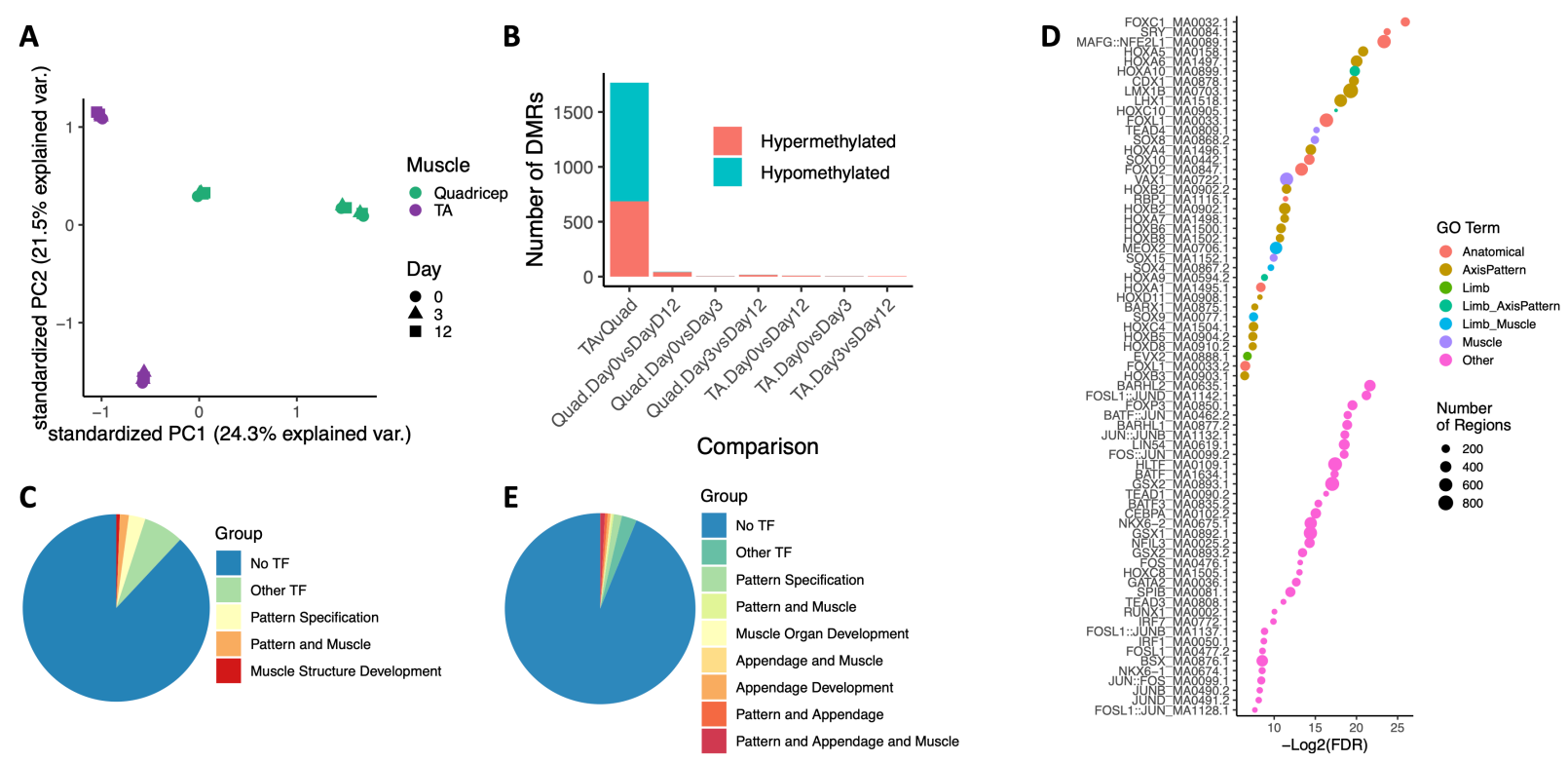

$\mathbf{F}$

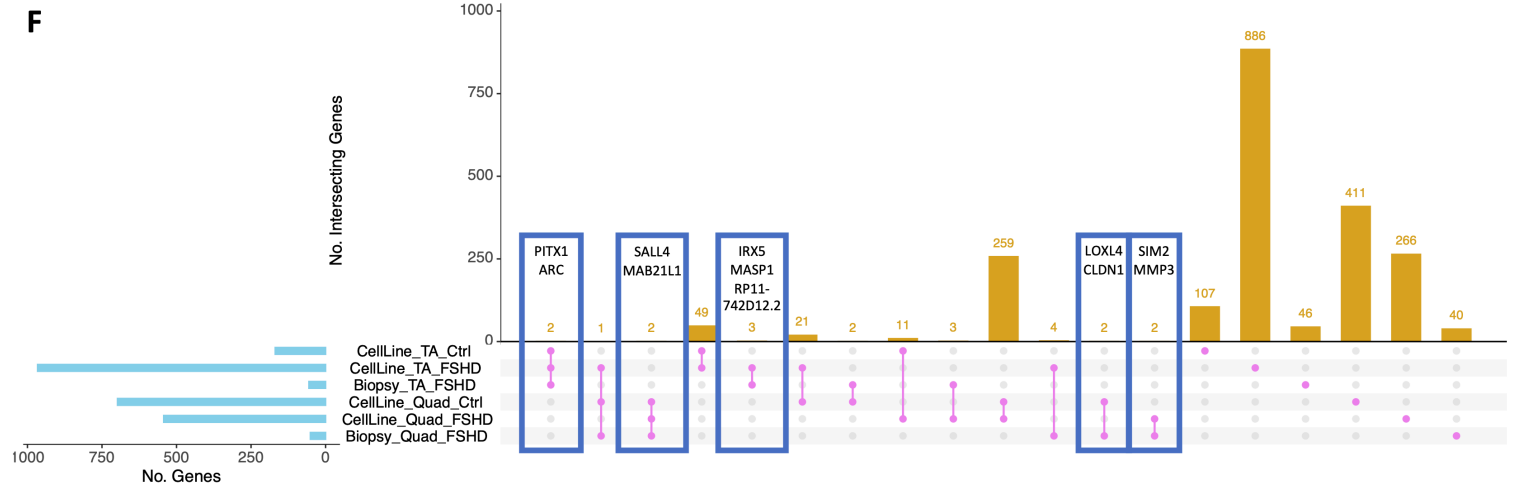

837 Figure S2: DNA methylation and gene expression differences for TA compared to

838 quadricep (A) Principal component analysis (PCA) of control TA and quadricep DNA

839 methylation. (B) Stacked barplot of the number of differentially methylated regions between TA

840 and quadricep. (C) Pie chart of the number of differentially methylated regions associated with

841 genes for transcription factors. Regions not associated with a transcription factor are in blue.

842 Regions associated with transcription factors are colored by annotated gene ontology terms for

843 pattern specification or muscle structure development. (D) Transcription factor motifs enriched

844 in differentially methylated regions between TA and quadricep. Color indicates the gene

845 ontology annotation associated with transcription factor, either anatomical development, axis

846 patterning, limb development, or muscle development (see Methods for specific GO terms). Size 
847 indicates number of regions with given motif. FDR cutoff of 0.05. (E) Pie chart of the number of

848 differentially expressed transcription factors for TA versus quadricep. Genes that are not

849 transcription factors are in blue. Transcription factors are colored by annotated gene ontology

850 terms for pattern specification, appendage development or muscle structure development. (F)

851 Upset plot of genes differentially expressed between TA and quadricep in control cells, FSHD2

852 cells or in FSHD biopsies from [26]. Number of genes higher in the given muscle in the given

853 comparison are indicated in the light blue barplot on the left. Number of genes found in the

854 intersections indicated in magenta are given in the gold barplot at top. Genes in intersections

855 outlined in blue are labelled. 
A

D
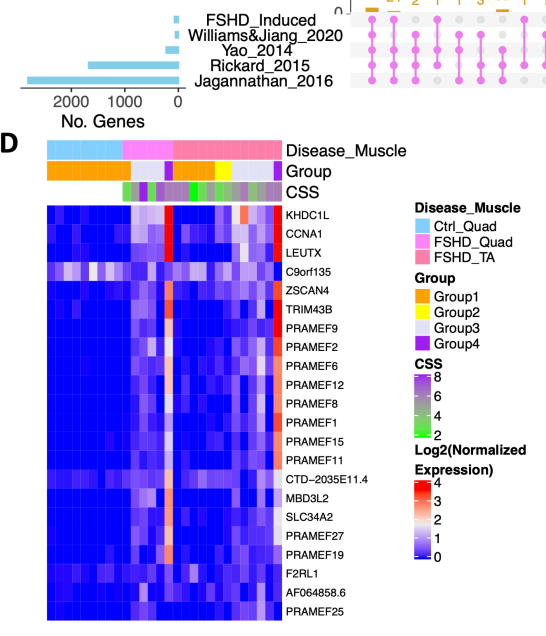
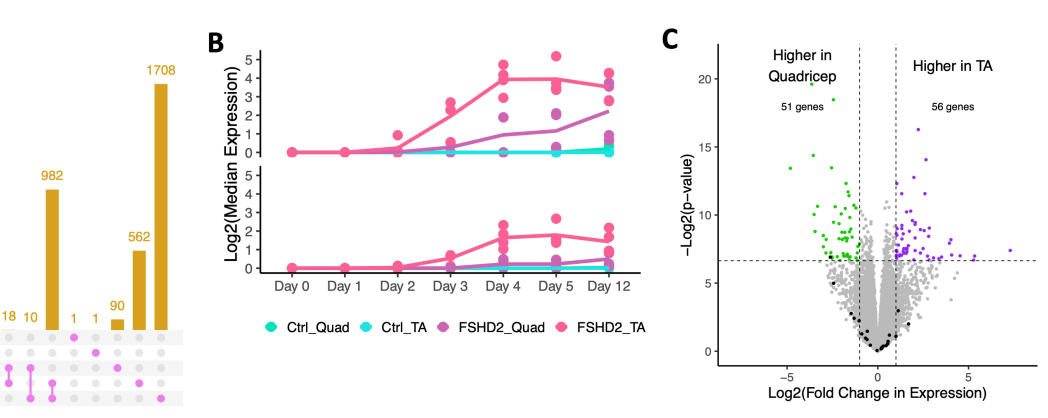

E

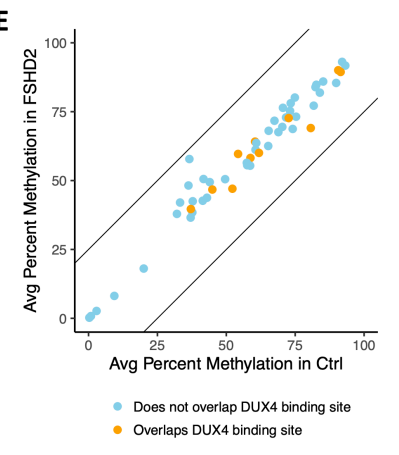

$\mathbf{F}$

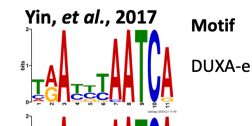

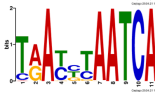

DUXA-EDBD-1

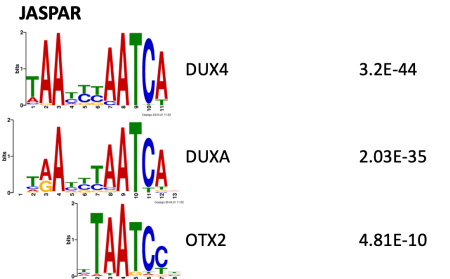

Figure S3: Assessment of FSHD-induced genes (A) Upset plot of FSHD-induced genes

compared with the gene list from [53] and reanalyzed data from $[21,75,76]$. Yao 2014 is a

comparison of differentiated FSHD and control myotubes [21]. Rickard 2015 is a comparison of

DUX4 expressing myocytes identified by a reporter to those negative for the reporter [76].

Jagannathan 2016 is a comparison of induced expression of DUX4 to non-induced [75]. (B)

Median expression for given groups of 74 FSHD-induced genes. Those identified in [53] are on

top, and those new from this analysis are on bottom. Dots represent median expression for

individual samples. Line represents the mean for the four samples in each group. (C) Volcano

plot of expression differences between TA and quadricep from FSHD biopsies from [26]. Genes

with higher expression in TA or quadricep are in purple and green, respectively. FSHD-induced

genes are colored in black. The number of genes higher in each muscle are labelled at the top.

(D) Heatmap of FSHD-induced gene expression detectable in control quadricep, FSHD

quadricep and FSHD TA biopsy samples from [26]. Groups were identified in [26] with an 
872 increase in group number correlating with an increase in DUX4 target gene expression. Clinical

873 severity score (CSS) is on a 10-point scale. (E) Scatterplot of percent methylation in the

874 promoters of FSHD-induced genes for control and FSHD2. Lines indicate a 25\% difference.

875 Promoters that overlap known DUX4 binding sites are colored orange. (F) Motifs from [35] or

876 [64] enriched in promoters of FSHD-induced genes. 
A

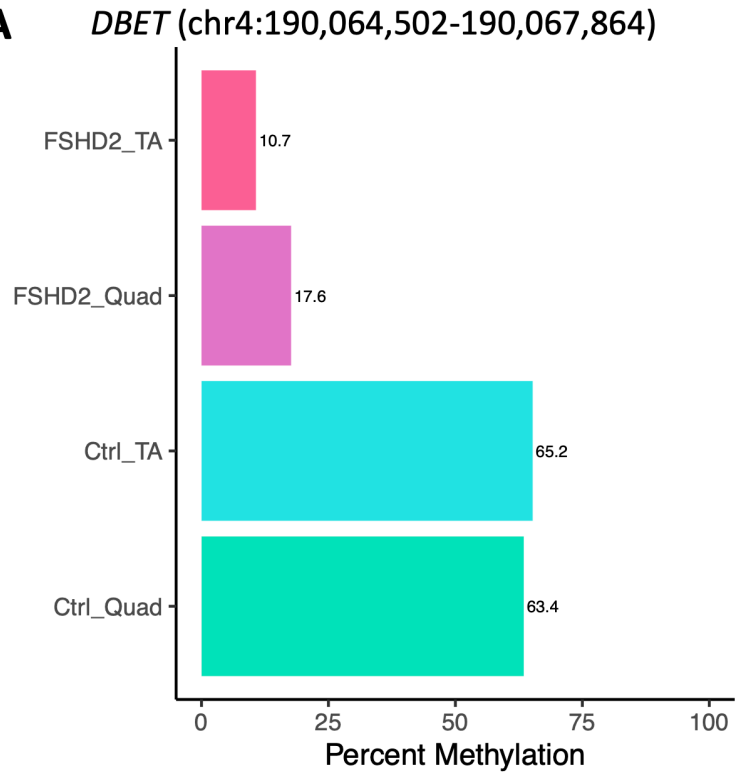

B

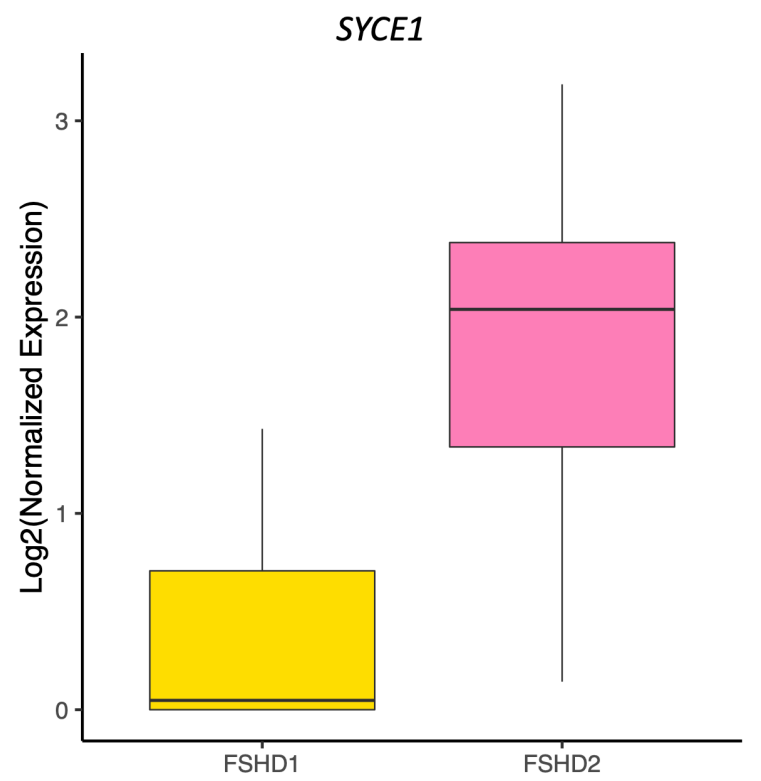

880 Figure S4: SMCHD1 related methylation and expression differences (A) Barplot of average

881 percent methylation near $D B E T$ on chromosome 4. Numbers to the left of bars give the average

882 percent methylation. (B) Boxplot of SYCE1 expression in FSHD1 and FSHD2 samples at day 0

883 of differentiation.

884 


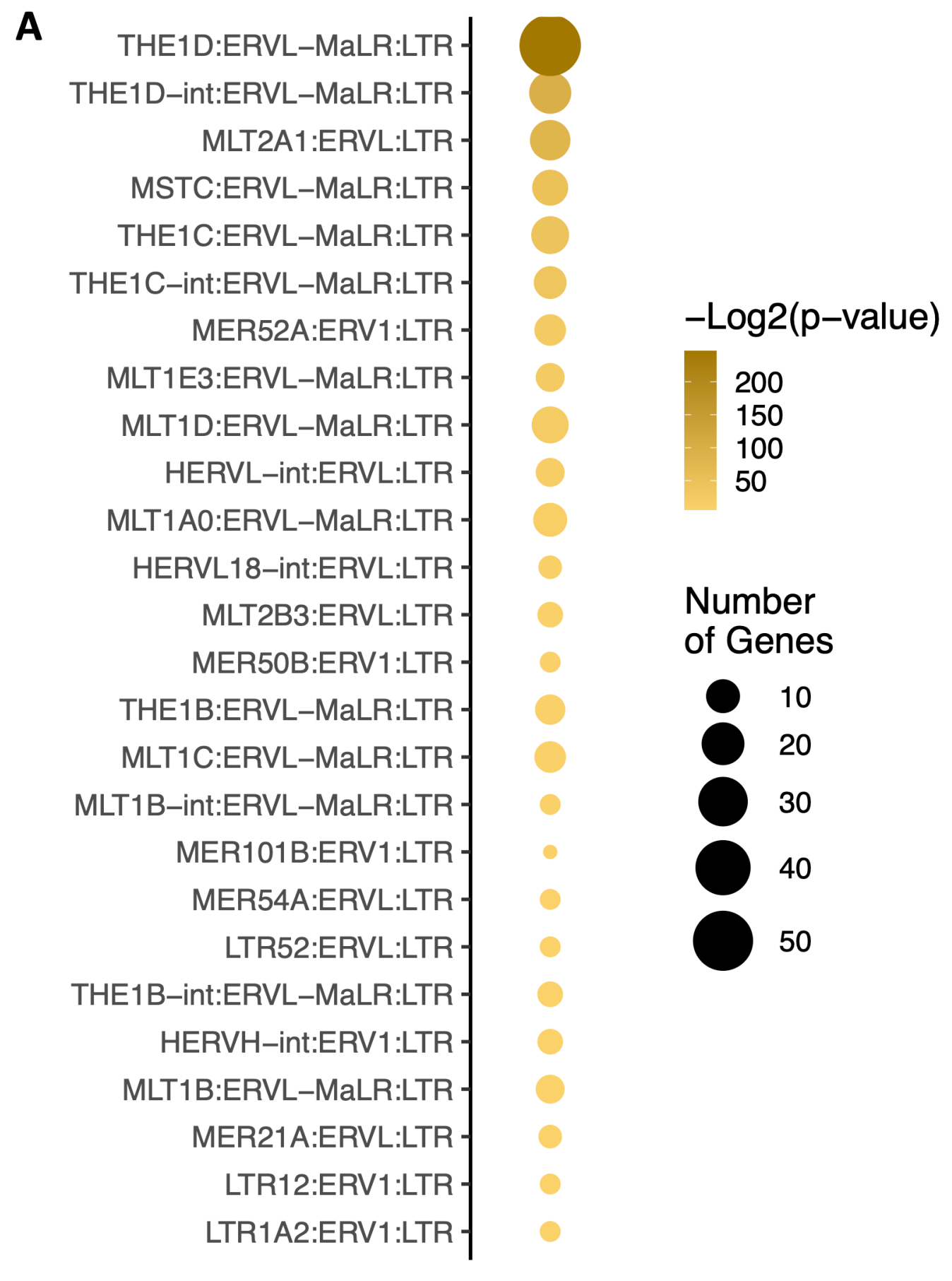

887 Figure S5: Enrichment of specific types of LTRs (A) Enrichment of specific types of LTRs upregulated in FSHD2. Classes with p-value $>0.05$ are not shown. Size indicates number of loci 889 in category. 


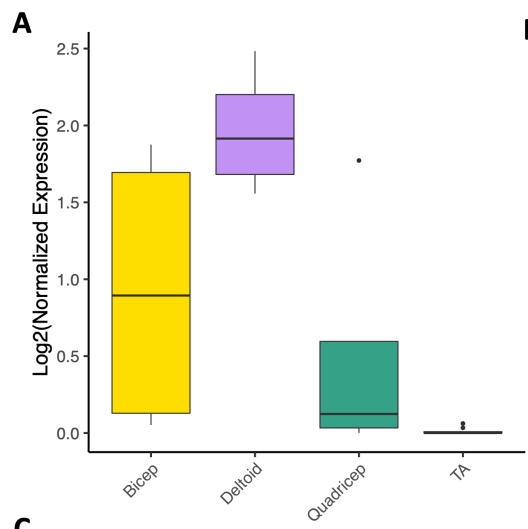

C

(

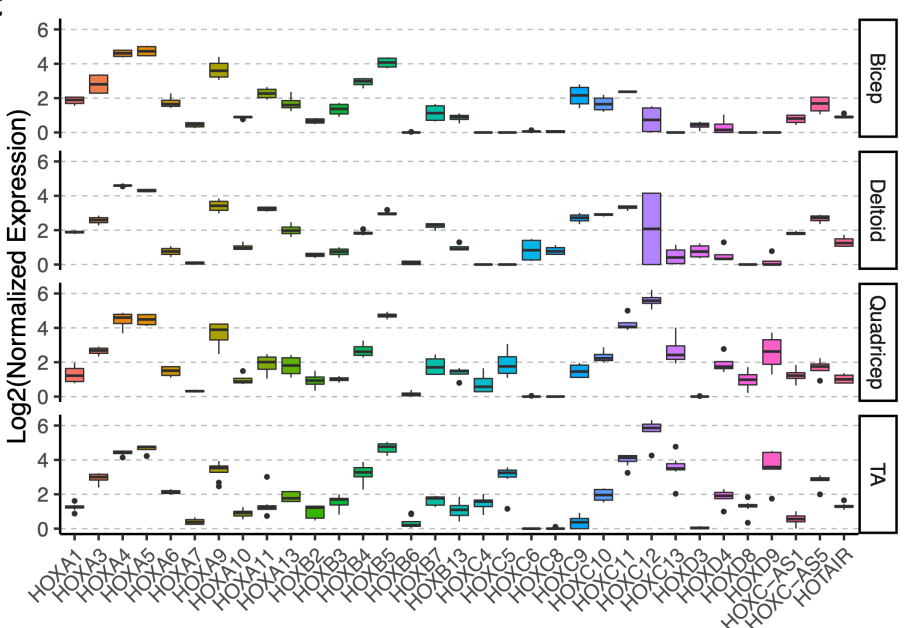

B

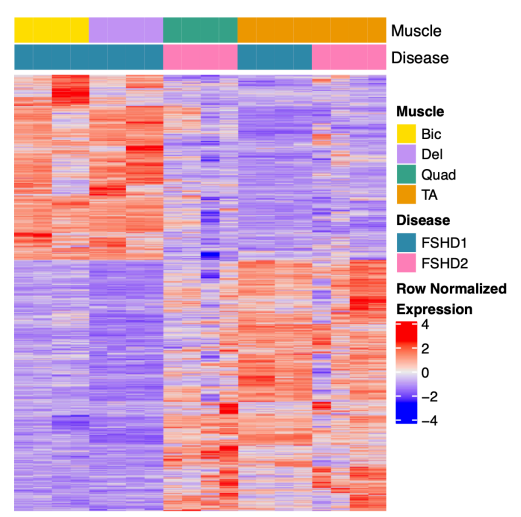

$\frac{m}{0}$

Figure S6: Muscle group specific expression (A) Boxplot of $T B X 5$ expression in the different

893 muscle groups at day 0 of differentiation. (B) Heatmap of genes specific to the upper (bicep and

894 deltoid) or lower (TA and quadricep) body muscles at day 0 of differentiation. (C) Boxplot of

895 HOX gene expression at day 0 of differentiation split by muscle group. (D) Heatmap of genes

896 with differential expression in highly (TA and bicep) and less (quadricep and deltoid) susceptible

897 muscle groups at days 0 and 5 of differentiation combined.

898 
900 Table S1: Differentially methylated regions between TA and quadricep in control cells

901 Genes are annotated by GREAT. Percent difference in methylation given in "meth.diff” column

902 with positive values indicating higher methylation in TA.

903

904 Table S2: Differentially expressed genes between TA and quadricep in control cells TFs are

905 labelled as well as TFs whose motifs were enriched in differentially methylated regions.

906

907 Table S3: Motifs enriched in promoters of FSHD-induced genes

908

909 Table S4: Differentially methylated regions between FSHD2 and control Genes are

910 annotated by GREAT. Percent difference in methylation given in "meth.diff" column with

911 positive values indicating higher methylation in FSHD2.

912

913 Table S5: Differentially expressed genes between FSHD2 and control

914

915 Table S6: 580 TE loci with increased expression in FSHD2 during myogenesis Loci that

916 overlap DUX4 binding sites of FSHD-induced genes are indicated in the "DUX4_BS" and

917 “OverlapFI” columns, respectively. Loci that overlap peaks from [24] are also indicated.

918

919 Table S7: Genes with expression differences for highly susceptible and less susceptible

920 muscle groups

921 